\title{
GLOBAL EXISTENCE AND BOUNDEDNESS FOR QUASI-VARIATIONAL SYSTEMS
}

\author{
GIANCARLO CANTARELLI
}

(Received 12 February 1997)

\begin{abstract}
We consider quasi-variational ordinary differential systems, which may be considered as the motion law for holonomic mechanical systems. Even when the potential energy of the system is not bounded from below, by constructing appropriate Liapunov functions and using the comparison method, we obtain sufficient conditions for global existence of solutions in the future and for their partial boundedness.
\end{abstract}

Keywords and phrases. Global existence, boundedness.

1991 Mathematics Subject Classification. 34C11, 70 H35.

1. Introduction. Let $G(u, p)$ and $F(t, u)$ be scalar functions and $Q(t, u, p)$ an Nvector $(N \geq 1)$. We consider vector solutions $u=\left(u_{1}, \ldots, u_{N}\right)$ of the quasi-variational ordinary differential system

$$
\frac{d}{d t}(\nabla G(u, \dot{u}))-\nabla_{u} G(u, \dot{u})+\nabla_{u} F(t, u)=Q(t, u, \dot{u}),
$$

where $d / d t$ and "." denote differentiation with respect to the independent variable $t \in \mathbb{R}^{+}=[0, \infty)$, and

$$
\nabla=\left(\frac{\partial}{\partial p_{1}}, \ldots, \frac{\partial}{\partial p_{N}}\right), \quad \nabla_{u}=\left(\frac{\partial}{\partial u_{1}}, \ldots, \frac{\partial}{\partial u_{N}}\right) .
$$

It is supposed throughout the paper that

(1) $G: \mathbb{R}^{N} \times \mathbb{R}^{N} \rightarrow \mathbb{R}^{+}$is a function of class $C^{1}$, strictly convex in the variable $p$ for every $u \in \mathbb{R}^{N}$, with $G(u, 0)=0$ and $\nabla G(u, 0)=0$ on $\mathbb{R}^{N}$;

(2) $F: \mathbb{R}^{+} \times \mathbb{R}^{N} \rightarrow \mathbb{R}$ is a function of class $C^{1}$;

(3) $Q: \mathbb{R}^{+} \times \mathbb{R}^{N} \times \mathbb{R}^{N} \rightarrow \mathbb{R}^{N}$ is a continuous vector function.

A function $u(t)$ defined on an interval $I \subset \mathbb{R}^{+}$is called a (weak) solution of (1.1) if $u(t)$ and $\nabla G(u(t), \dot{u}(t))$ are functions of class $C^{1}$ such that

$$
\frac{d}{d t}(\nabla G(u(t), \dot{u}(t)))-\nabla_{u} G(u(t), \dot{u}(t))+\nabla_{u} F(t, u(t))=Q(t, u(t), \dot{u}(t)) \quad \text { on } I .
$$

Let $H(u, p)$ be the Legendre transform in the variable $p$ of the function $G(u, p)$, namely,

$$
H(u, p)=(\nabla G(u, p), p)-G(u, p),
$$

where $(\cdot, \cdot)$ denotes the inner product in $\mathbb{R}^{N}$. For every fixed $u \in \mathbb{R}^{N}$, the function 
$H(u, p)$ is positive definite with respect to $p$. Moreover, in spite of the fact that neither $\dot{u}(t)$ nor $H(u, p)$ need to be separately differentiable, the composite function $H(u(t), \dot{u}(t))$ is differentiable along any solution $u(t)$ and we have [11, Thm. 8]

$$
\frac{d}{d t}[H(u(t), \dot{u}(t))+F(t, u(t))]=(Q(t, u(t), \dot{u}(t)), \dot{u}(t))+F_{t}(t, u(t)) \quad \text { on } I \text {. }
$$

In the present paper, we are concerned with global existence in the future and boundedness for solutions of the system (1.1). This problem was initiated by P. Pucci and J. Serrin in $[9,10]$. Later, in $[3,5]$ the author studied the same problem, applying and extending to the system (1.1) the method introduced by C. Risito in [12] in his study of the solutions of the Lagrange equations. Indeed, (1.1) may be considered as the motion equation for a holonomic dynamical system with $N$ degrees of freedom, whose Lagrangian is defined by an action energy $H$ and a potential energy $F$.

We emphasize that throughout the paper, we do not require the function $F$ to be bounded below in $I \times \mathbb{R}^{N}$, for any bounded interval $I \subset \mathbb{R}^{+}$. Such a condition, essential in $[9,12,6]$, is usually required when we take as Liapunov function the total energy $H+F$. For this reason, in the present paper, we use Liapunov functions obtained by perturbing the total energy.

We have divided the paper into two parts. The first part (Sections 2 and 3) is based on the hypothesis (2.2) on the function $H$. The second part (Sections 4 and 5) is based on the hypothesis (4.2), which is essentially a condition on vector $Q$. It is worth noting that, by disregarding the term $\mu(t) V+\rho(t) \psi(V)$, the right-hand side of (4.2) may be considered as the power of particular dissipative forces $Q$.

Many results of the papers $[9,10,3,5,12,6,2]$ are included here as special cases. In particular, Corollaries 2.2, 2.3, 4.1, and 4.3 show that the results of Sections 2 and 4 generalize the earlier conclusions in [5].

Throughout the paper, and without further mention, we denote by the symbols $x$ and $y$ the vectors formed with the first $i(1 \leq i \leq N)$ components of $u$ and $p$, respectively, i.e.,

$$
x=\left(u_{1}, \ldots, u_{i}\right), \quad y=\left(p_{1}, \ldots, p_{i}\right) .
$$

Moreover, the symbol $z$ denotes a $j$-vector $(1 \leq j \leq N)$ formed with $j$ components of $p$, namely,

$$
z=\left(p_{k_{1}}, \ldots, p_{k_{j}}\right),
$$

where $k_{1}, \ldots, k_{j}$ are integers such that $1 \leq k_{1}<\cdots<k_{j} \leq N$.

Finally, for each constant $\omega>0$, the symbol $\Omega$ denotes the set

$$
\Omega=\left\{u \in \mathbb{R}^{N}:|x| \leq \omega\right\},
$$

where $|\cdot|$ denotes the euclidean norm.

2. Global existence in the future, Part 1. The main hypotheses of Sections 2 and 3 follow

$$
\begin{array}{rlrl}
F(t, u) & \geq-f(\sigma(t) \tau(|x|)) & & \text { on } \mathbb{R}^{+} \times \mathbb{R}^{N}, \\
H(u, p) \geq h\left(\tau^{\prime}(|x|)|y|\right) & & \text { on } \mathbb{R}^{N} \times \mathbb{R}^{N},
\end{array}
$$


where

(a) $f: \mathbb{R}^{+} \rightarrow \mathbb{R}^{+}$is a function of class $C^{1}$, convex, strictly increasing and unbounded;

(b) $h: \mathbb{R}^{+} \rightarrow \mathbb{R}^{+}$is a continuous function, strictly increasing and unbounded with $h(0)=0$;

(c) $\sigma: \mathbb{R}^{+} \rightarrow(0, \infty)$ is a function of class $C^{1}$;

(d) $\tau: \mathbb{R}^{+} \rightarrow \mathbb{R}^{+}$is a function of class $C^{1}$, strictly increasing and unbounded with $\tau(0)=0$, and $\tau^{\prime}$ denotes its derivative.

Furthermore, we assume that, corresponding to each constant $\omega>0$, there exists a continuous, strictly increasing and unbounded function $\Phi_{\omega}$ such that

$$
H(u, p) \geq \Phi_{\omega}(|p|) \quad \text { on } \Omega \times \mathbb{R}^{N} .
$$

From (2.2), it follows immediately that condition (2.3) holds in the important case where $x=u$.

REMARK 2.1. A condition of the type (2.2) first appears in [12]. In fact, denoting by $q=\left(q_{1}, \ldots, q_{N}\right)$ the independent Lagrangian coordinates of a mechanical system with $N$ degrees of freedom, and by $T(q, \dot{q})$ its kinetic energy, C. Risito assumes that $[12$, Thm. 2]

$$
T(q, \dot{q}) \geq \alpha(|q|)|\dot{q}|^{2} \quad \text { on } \mathbb{R}^{N} \times \mathbb{R}^{N},
$$

where $\alpha: \mathbb{R}^{+} \rightarrow(0, \infty)$ is a continuous function such that $\int^{\infty} \sqrt{\alpha(s)} d s=\infty$. Clearly, condition (2.4) coincides with (2.2) when $x=q, h(s)=s^{2}$ and $\tau(s)=\int_{0}^{s} \sqrt{\alpha(r)} d r$.

Conditions (2.1) and (2.2) first appear together in [2] in the particular case where $f(s)=h(s)=s^{2}$.

REMARK 2.2. By assumption (a), both the derivative $f^{\prime}$ and the inverse $f^{-1}$ are increasing functions, and $f^{-1}$ is unbounded. Thus,

$$
\psi(s):=\left(f^{\prime} \circ f^{-1}\right)(s) f^{-1}(s), \quad\left(f^{\prime} \circ f^{-1}=f^{\prime}\left(f^{-1}\right)\right)
$$

is also an increasing and unbounded function.

In this section, and in the following one, we denote by $V=V(t, u, p)$ the Liapunov function

$$
V(t, u, p)=H(u, p)+F(t, u)+2 f(\sigma(t) \tau(|x|)) .
$$

Let $h^{-1}$ be the inverse of the function $h$.

THEOREM 2.1. Suppose the conditions (2.1), (2.2), and (2.3) hold. Moreover, assume that

(i) there exist three continuous functions $\mu, \rho: \mathbb{R}^{+} \rightarrow \mathbb{R}$ and $\phi: \mathbb{R}^{+} \rightarrow \mathbb{R}^{+}$such that $(Q(t, u, p), p)+F_{t}(t, u) \leq \mu(t) \phi(V)+\rho(t) \psi(V)$ on $\mathbb{R}^{+} \times \mathbb{R}^{N} \times \mathbb{R}^{N} ;$

(ii) $\int^{\infty} d s /\left(s+(\phi \circ f)(s) / f^{\prime}(s)+\left(h^{-1} \circ f\right)(s)\right)=\infty$.

Then all solutions of (1.1) exist globally in the future.

Proof. Suppose for contradiction that there exists a solution $u=u(t)$ of (1.1) defined on a right maximal interval of existence $\left[t_{0}, T\right)$, with $T<\infty$, i.e., $u$ cannot be 
continued to the right beyond $T$. Then, as is well known, we have

$$
\lim _{t \rightarrow T^{-}}\{|u(t)|+|\dot{u}(t)|\}=\infty .
$$

We, now, divide the proof into two parts.

(I) For every $t \in\left[t_{0}, T\right)$, we put

$$
v(t)=V(t, u(t), \dot{u}(t)) .
$$

We claim that $v(t)$ is unbounded on $\left[t_{0}, T\right)$, i.e.,

$$
\limsup _{t \rightarrow T^{-}} v(t)=\infty .
$$

Otherwise, there exists a constant $\gamma$ such that

$$
v(t) \leq \gamma \quad \text { on }\left[t_{0}, T\right) .
$$

In view of (2.1), it is

$$
f(\sigma(t) \tau(|x(t)|)) \leq v(t) \text { on }\left[t_{0}, T\right),
$$

from which follows, putting $\sigma_{*}=\min \left\{\sigma(t): t_{0} \leq t \leq T\right\}$ and taking into account (2.10),

$$
\tau(|x(t)|) \leq \frac{f^{-1}(\gamma)}{\sigma_{*}} \quad \text { on }\left[t_{0}, T\right) .
$$

Thus, letting $\omega=\tau^{-1}\left(f^{-1}(\gamma) / \sigma_{*}\right)$, we see that $u(t) \in \Omega$ for every $t \in\left[t_{0}, T\right)$. Hence, by (2.1) and (2.3), it follows that

$$
\Phi_{\omega}(|\dot{u}(t)|) \leq H(u(t), \dot{u}(t)) \leq v(t) \quad \text { on }\left[t_{0}, T\right),
$$

and, in virtue of (2.10),

$$
|\dot{u}(t)| \leq \Phi_{\omega}^{-1}(\gamma) \quad \text { on }\left[t_{0}, T\right) .
$$

Finally, taking into account the fact that $(d / d t)|u(t)| \leq|\dot{u}(t)|$ and integrating with respect to $t$ on $\left[t_{0}, T\right)$, we get

$$
|u(t)| \leq\left|u\left(t_{0}\right)\right|+\Phi_{\omega}^{-1}(\gamma)\left(T-t_{0}\right) \quad \text { on }\left[t_{0}, T\right) .
$$

Clearly, (2.14) and (2.15) contradict (2.7). This contradiction completes the proof of the claim.

(II) In order to obtain a contradiction with (2.9), let us consider the derivative of the function $v(t)$. By identity (1.5) and condition (i), we have

$$
\begin{aligned}
\dot{v} & =(Q(t, u, \dot{u}), \dot{u})+F_{t}(t, u)+2 \frac{d}{d t} f(\sigma \tau(|x|)) \\
& \leq \mu \phi(v)+\rho \psi(v)+2 f^{\prime}(\sigma \tau(|x|))\left[\dot{\sigma} \tau(|x|)+\sigma \frac{d}{d t} \tau(|x|)\right] \quad \text { on }\left[t_{0}, T\right) .
\end{aligned}
$$


Since $(d / d t)|x(t)| \leq|\dot{x}(t)|$, by (2.2), we obtain

$$
\frac{d}{d t} \tau(|x(t)|) \leq \tau^{\prime}(|x(t)|)|\dot{x}(t)| \leq h^{-1}(v(t)) \quad \text { on }\left[t_{0}, T\right) .
$$

Moreover, condition (2.1) implies that

$$
\sigma(t) \tau(|x(t)|) \leq f^{-1}(v(t)) \quad \text { on }\left[t_{0}, T\right),
$$

so that we get

$$
f^{\prime}(\sigma(t) \tau(|x(t)|)) \leq\left(f^{\prime} \circ f^{-1}\right)(v(t)) \text { on }\left[t_{0}, T\right),
$$

since $f^{\prime}$ is an increasing function.

By virtue of (2.17), (2.18), and (2.19), from (2.16), we thus obtain

$$
\begin{aligned}
\dot{v} \leq & \mu \phi(v)+\left(\rho+2 \frac{|\dot{\sigma}|}{\sigma}\right)\left(f^{\prime} \circ f^{-1}\right)(v) f^{-1}(v) \\
& +2 \sigma\left(f^{\prime} \circ f^{-1}\right)(v) h^{-1}(v) \quad \text { on }\left[t_{0}, T\right),
\end{aligned}
$$

and so

$$
\dot{v} \leq c(t)\left[\phi(v)+\left(f^{\prime} \circ f^{-1}\right)(v) f^{-1}(v)+\left(f^{\prime} \circ f^{-1}\right)(v) h^{-1}(v)\right] \quad \text { on }\left[t_{0}, T\right)
$$

provided that

$$
c(t)=\max _{t \in \mathbb{R}^{+}}\left\{\mu(t), \rho(t)+2 \frac{|\dot{\sigma}(t)|}{\sigma(t)}, 2 \sigma(t)\right\} .
$$

We now consider the comparison equation

$$
\dot{w}=c(t)\left[\phi(w)+\left(f^{\prime} \circ f^{-1}\right)(w) f^{-1}(w)+\left(f^{\prime} \circ f^{-1}\right)(w) h^{-1}(w)\right] .
$$

Let $a, b$ be real positive constants. Since

$$
\begin{aligned}
\int_{a}^{b} \frac{d s}{s+(\phi \circ f)(s) / f^{\prime}(s)+\left(h^{-1} \circ f\right)(s)} \\
\quad=\int_{f(a)}^{f(b)} \frac{d s}{\phi(s)+\left(f^{\prime} \circ f^{-1}\right)(s) f^{-1}(s)+\left(f^{\prime} \circ f^{-1}\right)(s) h^{-1}(s)},
\end{aligned}
$$

and since $f(s) \rightarrow \infty$ as $s \rightarrow \infty$, by virtue of condition (ii), we get

$$
\int^{\infty} \frac{d s}{\phi(s)+\left(f^{\prime} \circ f^{-1}\right)(s) f^{-1}(s)+\left(f^{\prime} \circ f^{-1}\right)(s) h^{-1}(s)}=\infty .
$$

This implies that all the solutions of the comparison equation (2.23) exist globally in the future [7].

Let $w(t)$ be a solution of (2.23) satisfying the initial condition

$$
w\left(t_{0}\right) \geq v\left(t_{0}\right)=H\left(u_{0}, \dot{u}_{0}\right)+F\left(t_{0}, u_{0}\right)+2 f\left(\sigma\left(t_{0}\right) \tau\left(\left|x_{0}\right|\right)\right),
$$

where $u_{0}=u\left(t_{0}\right), \dot{u}_{0}=\dot{u}\left(t_{0}\right)$, and $x_{0}=x\left(t_{0}\right)$. By the comparison method, it follows that [8]

$$
v(t) \leq w(t) \text { on }\left[t_{0}, T\right) .
$$

This contradicts (2.9) since $w(t)$ is a continuous function defined on $\left[t_{0}, \infty\right)$, and completes the proof. 
Theorem 2.1 has the following corollary, which coincides with [5, Thm. 1].

COROLLARY 2.1. Suppose that condition (2.3) holds. Moreover, assume that

$$
\begin{gathered}
H(u, p) \geq\left(\tau^{\prime}(|x|)|y|\right)^{m} \quad \text { on } \mathbb{R}^{N} \times \mathbb{R}^{N}, \\
F(t, u) \geq-(\sigma(t) \tau(|x|))^{n} \quad \text { on } \mathbb{R}^{+} \times \mathbb{R}^{N}, \\
(Q(t, u, p), p)+F_{t}(t, u) \\
\leq v(t)\left[\left(\tau^{\prime}(|x|)|y|\right)^{m}+(\sigma(t) \tau(|x|))^{n}\right] \quad \text { on } \mathbb{R}^{+} \times \mathbb{R}^{N} \times \mathbb{R}^{N},
\end{gathered}
$$

where $v: \mathbb{R}^{+} \rightarrow \mathbb{R}^{+}$is a continuous function, and $1 \leq n \leq m$ are real constants.

Then all the solutions of (1.1) exist globally in the future.

Proof. It is easy to see that all the conditions of Theorem 2.1 are satisfied by choosing $f(s)=s^{n}, h(s)=s^{m}, \mu(t)=0$, and $\rho(t)=v(t) / n$.

REMARK 2.3. By strengthening condition (i) of Theorem 2.1, we can weaken condition (ii). It is easy to show that condition (i) and (ii) of Theorem 2.1 can be replaced by the following ones

$$
\begin{gathered}
(Q(t, u, p), p)+F_{t}(t, u) \leq \rho(t) \psi(V) \quad \text { on } \mathbb{R}^{+} \times \mathbb{R}^{N} \times \mathbb{R}^{N}, \\
\int^{\infty} \frac{d s}{s+\left(h^{-1} \circ f\right)(s)}=\infty,
\end{gathered}
$$

or

$$
\begin{gathered}
(Q(t, u, p), p)+F_{t}(t, u) \leq-2 \dot{\sigma}(t) \tau(|x|) f^{\prime}(\sigma(t) \tau(|x|)) \quad \text { on } \mathbb{R}^{+} \times \mathbb{R}^{N} \times \mathbb{R}^{N}, \\
\int^{\infty} \frac{d s}{\left(h^{-1} \circ f\right)(s)}=\infty .
\end{gathered}
$$

Observe that (2.33) is stronger than (2.31). In fact, keeping (2.18) and (2.19) in mind, we see that

$$
\begin{aligned}
-2 \dot{\sigma}(t) \tau & (|x|) f^{\prime}(\sigma(t) \tau(|x|)) \\
& \leq 2 \frac{|\dot{\sigma}(t)|}{\sigma(t)}\left(f^{\prime} \circ f^{-1}\right)(V) f^{-1}(V) \text { on } \mathbb{R}^{+} \times \mathbb{R}^{N} \times \mathbb{R}^{N},
\end{aligned}
$$

so that (2.31) is satisfied with $\rho(t)=2|\dot{\sigma}(t)| / \sigma(t)$.

In the rest of the section, we assume that

$$
\int^{\infty} \frac{d s}{\left(h^{-1} \circ f\right)(s)}<\infty .
$$

This condition is said to be critical for the system (1.1) because neither condition (ii) of Theorem 2.1 nor the weaker conditions (2.32), (2.34) can then be satisfied. Thus, in this case, we are unable to ensure the global existence in the future of all the solutions of system (1.1).

In order to ensure global existence in the future at least of submanifolds of solutions, we consider two cases. 
CASE 1. Assume that there exists a continuous function $\mu: \mathbb{R}^{+} \rightarrow \mathbb{R}$ such that

$$
\begin{aligned}
& (Q(t, u, p), p)+F_{t}(t, u) \\
& \quad \leq \mu(t) V-2 \dot{\sigma}(t) \tau(|x|) f^{\prime}(\sigma(t) \tau(|x|)) \quad \text { on } \mathbb{R}^{+} \times \mathbb{R}^{N} \times \mathbb{R}^{N},
\end{aligned}
$$

and denote by $J(M) \subset \mathbb{R}^{+}$the range of the function

$$
M(t)=\exp \left\{-\int_{0}^{t} \mu(s) d s\right\} .
$$

Fix $t_{0} \geq 0$ and put $M_{0}=M\left(t_{0}\right)$.

ThEOREM 2.2. Suppose that conditions (2.1), (2.2), (2.3), and (2.37) hold. Moreover, assume that there exist two continuous functions $\alpha: \mathbb{R}^{+} \rightarrow \mathbb{R}^{+}$and $\beta: J(M) \rightarrow(0, \infty)$, and a real constant $a_{0} \geq M_{0} f(0)$, such that

$$
\begin{gathered}
\left(f^{\prime} \circ f^{-1}\right)(s) h^{-1}(s) \leq \frac{\alpha(r s)}{\beta(r)} \quad \text { on } J(M) \times[f(0), \infty), \\
2 \int_{t_{0}}^{\infty} \frac{\sigma(t) M(t)}{\beta(M(t))} d t=\int_{a_{0}}^{\infty} \frac{d s}{\alpha(s)} .
\end{gathered}
$$

Then the solutions $u\left(t, t_{0}, u_{0}, \dot{u}_{0}\right)$ of (1.1) such that $V\left(t_{0}, u_{0}, \dot{u}_{0}\right) \leq a_{0} / M_{0}$ exist globally in the future.

Proof. Conditions of the type (2.39) and (2.40) have been introduced by the author in [4]. We prove the theorem by the methods of [4, Thm. 1].

Let $u(t)=u\left(t, t_{0}, u_{0}, \dot{u}_{0}\right)$ be a solution of (1.1) with $v_{0}=V\left(t_{0}, u_{0}, \dot{u}_{0}\right) \leq a_{0} / M_{0}$. Suppose for contradiction that $u(t)$ is defined on a right maximal interval of existence $\left[t_{0}, T\right)$ with $T<\infty$. If $v(t)=V(t, u(t), \dot{u}(t))$, then (2.9) holds.

Repeating the second part of the proof of Theorem 2.1 with $\phi(s)=s$, we obtain the following differential inequality, which replaces (2.20),

$$
\dot{v}(t) \leq \mu(t) v(t)+2 \sigma(t)\left(f^{\prime} \circ f^{-1}\right)(v(t)) h^{-1}(v(t)) \text { on }\left[t_{0}, T\right) .
$$

For every $t \in\left[t_{0}, T\right)$, put

$$
q(t)=M(t) v(t)
$$

where $M(t)$ is given by (2.38). Since

$$
\dot{q}(t)=-\mu(t) M(t) v(t)+M(t) \dot{v}(t) \quad \text { on }\left[t_{0}, T\right),
$$

in view of (2.41), it follows that

$$
\dot{q}(t) \leq 2 \sigma(t) M(t)\left(f^{\prime} \circ f^{-1}\right)(v(t)) h^{-1}(v(t)) \text { on }\left[t_{0}, T\right) .
$$

By using condition (2.39) with $r=M(t)$ and $s=v(t)$, we obtain

$$
\left(f^{\prime} \circ f^{-1}\right)(v(t)) h^{-1}(v(t)) \leq \frac{\alpha(q(t))}{\beta(M(t))} \quad \text { on }\left[t_{0}, T\right) .
$$


Hence, from (2.44),

$$
\dot{q}(t) \leq 2 \frac{\sigma(t) M(t)}{\beta(M(t))} \alpha(q(t)) \quad \text { on }\left[t_{0}, T\right)
$$

Consider the comparison equation

$$
\dot{w}=2 \frac{\sigma(t) M(t)}{\beta(M(t))} \alpha(w)
$$

Since $q\left(t_{0}\right)=M_{0} v_{0} \leq a_{0}$, condition (2.40) implies that

$$
2 \int_{t_{0}}^{\infty} \frac{\sigma(t) M(t)}{\beta(M(t))} d t \leq \int_{M_{0} v_{0}}^{\infty} \frac{d s}{\alpha(s)},
$$

and this ensures that the solution $w(t)=w\left(t, t_{0}, M_{0} v_{0}\right)$ of (2.47) exists globally in the future [4]. Since $w\left(t_{0}\right)=q\left(t_{0}\right)$, by the comparison method, we have

$$
q(t) \leq w(t) \text { on }\left[t_{0}, T\right),
$$

and so

$$
v(t) \leq \frac{w(t)}{M(t)} \quad \text { on }\left[t_{0}, T\right) .
$$

Since $w(t) / M(t)$ is a continuous function defined on $\left[t_{0}, \infty\right),(2.50)$ contradicts (2.9). This completes the proof.

CASE 2. Assume that there exists a continuous function $\rho: \mathbb{R}^{+} \rightarrow \mathbb{R}$ such that

$$
\begin{aligned}
& (Q(t, u, p), p)+F_{t}(t, u) \\
& \leq \rho(t) \psi(V)-2 \dot{\sigma}(t) \tau(|x|) f^{\prime}(\sigma(t) \tau(|x|)) \quad \text { on } \mathbb{R}^{+} \times \mathbb{R}^{N} \times \mathbb{R}^{N},
\end{aligned}
$$

and denote by $J(P) \subset \mathbb{R}^{+}$the range of the function

$$
P(t)=\exp \left\{-\int_{0}^{t} \rho(s) d s\right\} .
$$

Fix $t_{0} \geq 0$ and put $P_{0}=P\left(t_{0}\right)$.

Theorem 2.3. Suppose that conditions (2.1), (2.2), (2.3), and (2.51) hold. Moreover, assume that there exist two continuous functions $\tilde{\alpha}: \mathbb{R}^{+} \rightarrow \mathbb{R}^{+}$and $\tilde{\beta}: J(P) \rightarrow(0, \infty)$, and a real constant $b_{0} \geq 0$, such that

$$
\begin{gathered}
\left(h^{-1} \circ f\right)(s) \leq \frac{\tilde{\alpha}(r s)}{\tilde{\beta}(r)} \quad \text { on } J(P) \times \mathbb{R}^{+}, \\
2 \int_{t_{0}}^{\infty} \frac{\sigma(t) P(t)}{\tilde{\beta}(P(t))} d t=\int_{b_{0}}^{\infty} \frac{d s}{\tilde{\alpha}(s)} .
\end{gathered}
$$

Then the solutions $u\left(t, t_{0}, u_{0}, \dot{u}_{0}\right)$ of (1.1) such that $V\left(t_{0}, u_{0}, \dot{u}_{0}\right) \leq f\left(b_{0} / P_{0}\right)$ exist globally in the future.

Proof. Suppose for contradiction that a solution $u(t)=u\left(t, t_{0}, u_{0}, \dot{u}_{0}\right)$, with $v_{0}=$ $V\left(t_{0}, u_{0}, \dot{u}_{0}\right) \leq f\left(b_{0} / P_{0}\right)$, is defined on a right maximal interval of existence $\left[t_{0}, T\right)$, with $T<\infty$. If $v(t)=V(t, u(t), \dot{u}(t))$, then (2.9) holds. 
Repeating the second part of the proof of Theorem 2.1, in place of (2.20), we obtain

$$
\dot{v}(t) \leq\left(f^{\prime} \circ f^{-1}\right)(v(t))\left[\rho(t) f^{-1}(v(t))+2 \sigma(t) h^{-1}(v(t))\right] \quad \text { on }\left[t_{0}, T\right) .
$$

Denote, now, by $q(t)$ the function

$$
q(t)=P(t) f^{-1}(v(t)) \text { on }\left[t_{0}, T\right) .
$$

Since

$$
\dot{q}(t)=-\rho(t) P(t) f^{-1}(v(t))+P(t) \frac{\dot{v}(t)}{\left(f^{\prime} \circ f^{-1}\right)(v(t))} \quad \text { on }\left[t_{0}, T\right),
$$

from (2.55) follows

$$
\dot{q}(t) \leq 2 \sigma(t) P(t) h^{-1}(v(t)) \text { on }\left[t_{0}, T\right) .
$$

On the other hand, condition (2.53) implies that

$$
h^{-1}(v(t))=\left(h^{-1} \circ f\right)\left(f^{-1}(v(t))\right) \leq \frac{\tilde{\alpha}(q(t))}{\tilde{\beta}(P(t))} \quad \text { on }\left[t_{0}, T\right) .
$$

Hence, from (2.58),

$$
\dot{q}(t) \leq 2 \frac{\sigma(t) P(t)}{\tilde{\beta}(P(t))} \tilde{\alpha}(q(t)) \quad \text { on }\left[t_{0}, T\right) .
$$

Consider the comparison equation

$$
\dot{w}=2 \frac{\sigma(t) P(t)}{\tilde{\beta}(P(t))} \tilde{\alpha}(w),
$$

and put $w(t)=w\left(t, t_{0}, q_{0}\right)$, where $q_{0}=q\left(t_{0}\right)=P_{0} f^{-1}\left(v_{0}\right)$. Since $P_{0} f^{-1}\left(v_{0}\right) \leq b_{0}$, condition (2.54) gives

$$
2 \int_{t_{0}}^{\infty} \frac{\sigma(t) P(t)}{\tilde{\beta}(P(t))} d t \leq \int_{q_{0}}^{\infty} \frac{d s}{\tilde{\alpha}(s)},
$$

which ensures that the solution $w(t)$ exists globally in the future.

The rest of the proof follows exactly as before.

The following corollaries are helpful in applications. It is worth noting that they are corollaries of both Theorem 2.2 and Theorem 2.3.

COROLlary 2.2. Fix $t_{0} \geq 0$ and assume that $2 \int_{t_{0}}^{\infty} \sigma(t) d t \leq \int_{0}^{\infty} d s /\left(\left(h^{-1} \circ f\right)(s)\right)$. Moreover, suppose that conditions (2.1), (2.2), and (2.3) hold, together with

$$
(Q(t, u, p), p)+F_{t}(t, u) \leq-2 \dot{\sigma}(t) \tau(|x|) f^{\prime}(\sigma(t) \tau(|x|)) \quad \text { on } \mathbb{R}^{+} \times \mathbb{R}^{N} \times \mathbb{R}^{N} .
$$

If $r_{0} \geq 0$ is the real constant satisfying

$$
2 \int_{t_{0}}^{\infty} \sigma(t) d t=\int_{r_{0}}^{\infty} \frac{d s}{\left(h^{-1} \circ f\right)(s)}
$$

then the solutions $u\left(t, t_{0}, u_{0}, \dot{u}_{0}\right)$ of (1.1) such that $V\left(t_{0}, u_{0}, \dot{u}_{0}\right) \leq f\left(r_{0}\right)$ exist globally in the future. 
Proof. Since (2.37) holds with $\mu(t) \equiv 0$, we have $J(M)=[1]$. Thus, condition (2.39) of Theorem 2.2 is satisfied by

$$
\beta(s)=1 \quad \text { and } \quad \alpha(s)=\left(f^{\prime} \circ f^{-1}\right)(s) h^{-1}(s) .
$$

Therefore, as

$$
\int_{r_{0}}^{\infty} \frac{d s}{\left(h^{-1} \circ f\right)(s)}=\int_{f\left(r_{0}\right)}^{\infty} \frac{d s}{\left(f^{\prime} \circ f^{-1}\right)(s) h^{-1}(s)},
$$

we see that condition (2.40) of Theorem 2.2, with $M(t) \equiv 1$ and $a_{0}=f\left(r_{0}\right)$, coincides with (2.64).

Moreover also (2.51) holds by $\rho(t) \equiv 0$. This means that $J(P)=[1]$, and condition (2.53) of Theorem 2.3 is satisfied if

$$
\tilde{\beta}(s)=1 \quad \text { and } \quad \tilde{\alpha}(s)=\left(h^{-1} \circ f\right)(s) \quad \text { on } \mathbb{R}^{+} .
$$

Then we see, at once, that condition (2.54) of Theorem 2.3, where $P(t) \equiv 1$ and $b_{0}=r_{0}$, coincides with (2.64).

COROLLARY 2.3. Let $n>m \geq 1$ be real constants. Suppose that condition (2.3) holds together with the following:

$$
\begin{array}{cc}
H(u, p) \geq\left(\tau^{\prime}(|x|)|y|\right)^{m} & \text { on } \mathbb{R}^{N} \times \mathbb{R}^{N}, \\
F(t, u) \geq-(\sigma(t) \tau(|x|))^{n} & \text { on } \mathbb{R}^{+} \times \mathbb{R}^{N} .
\end{array}
$$

Moreover, let $\rho: \mathbb{R}^{+} \rightarrow \mathbb{R}^{+}$be a continuous function, and $P(t)=\exp \left\{-\int_{0}^{t} \rho(s) d s\right\}$. Putting $\lambda=(n-m) / m$, assume $\sigma P^{-\lambda} \in L^{1}\left(\mathbb{R}^{+}\right)$and

$$
\begin{aligned}
(Q(t, u, p), p)+F_{t}(t, u) \leq & n \rho(t)\left[\left(\tau^{\prime}(|x|)|y|\right)^{m}+(\sigma(t) \tau(|x|))^{n}\right] \\
& -2 n \frac{\dot{\sigma}(t)}{\sigma(t)}(\sigma(t) \tau(|x|))^{n} \quad \text { on } \mathbb{R}^{+} \times \mathbb{R}^{N} \times \mathbb{R}^{N} .
\end{aligned}
$$

Then the solutions $u\left(t, t_{0}, u_{0}, \dot{u}_{0}\right)$ of (1.1) such that

$$
H\left(u_{0}, \dot{u}_{0}\right)+F\left(t_{0}, u_{0}\right)+2\left(\sigma\left(t_{0}\right) \tau\left(\left|x_{0}\right|\right)\right)^{n} \leq P_{0}^{-n}\left[2 \lambda \int_{t_{0}}^{\infty} \sigma(t)(P(t))^{-\lambda} d t\right]^{-n / \lambda}
$$

exists globally in the future.

Proof. Conditions (2.1) and (2.2) are satisfied with $f(s)=s^{n}$ and $h(s)=s^{m}$, respectively. Thus, since $\psi(s)=n s$, condition (2.70) is equivalent to (2.51). Moreover, as

$$
\left(h^{-1} \circ f\right)(s)=s^{n / m}=s^{1+\lambda},
$$

we see that condition (2.53) of Theorem 2.3 is satisfied by choosing

$$
\tilde{\alpha}(s)=\tilde{\beta}(s)=s^{1+\lambda} .
$$


Then condition (2.54) of Theorem 2.3 becomes

$$
2 \int_{t_{0}}^{\infty} \frac{\sigma(t)}{(P(t))^{\lambda}} d t=\int_{b_{0}}^{\infty} \frac{d s}{s^{1+\lambda}} .
$$

Hence,

$$
b_{0}=\left[2 \lambda \int_{t_{0}}^{\infty} \frac{\sigma(t)}{(P(t))^{\lambda}} d t\right]^{-1 / \lambda} .
$$

Thus, the inequality (2.71) is equivalent to $V\left(t_{0}, u_{0}, \dot{u}_{0}\right) \leq\left(b_{0} / P_{0}\right)^{n}=f\left(b_{0} / P_{0}\right)$. This means that Corollary 2.3 is a corollary of Theorem 2.3.

In the same way, we show that Corollary 2.3 is a corollary of Theorem 2.2

REMARK 2.4. Conditions (2.40) and (2.54) of Theorems 2.2 and 2.3 can be satisfied only if

$$
\frac{\sigma M}{\beta(M)} \in L^{1}\left(\mathbb{R}^{+}\right) \quad \text { and } \quad \frac{\sigma P}{\tilde{\beta}(P)} \in L^{1}\left(\mathbb{R}^{+}\right)
$$

respectively.

In fact, since $1 \in J(M)$, putting $r=1$ in condition (2.39) of Theorem 2.2, we have

$$
\left(f^{\prime} \circ f^{-1}\right)(s) h^{-1}(s) \leq \frac{\alpha(s)}{\beta(1)} \text { on }[f(0), \infty),
$$

where $\beta(1)$ is a positive constant. We, thus, see that, for any $a \geq 0$ and $b \geq 0$,

$$
\int_{f(a)}^{f(b)} \frac{d s}{\alpha(s)} \leq \beta(1) \int_{f(a)}^{f(b)} \frac{d s}{\left(f^{\prime} \circ f^{-1}\right)(s) h^{-1}(s)}=\beta(1) \int_{a}^{b} \frac{d s}{\left(h^{-1} \circ f\right)(s)} .
$$

Hence, in view of (2.36),

$$
\int^{\infty} \frac{d s}{\alpha(s)}<\infty
$$

Likewise, we show that

$$
\int^{\infty} \frac{d s}{\tilde{\alpha}(s)}<\infty
$$

3. Boundedness, Part 1. In this section, we obtain sufficient conditions for global existence in the future and for partial boundedness of the solutions of (1.1). To this end, in addition to (2.2) and (2.3), we assume that the function $H(u, p)$ satisfies the future condition

$$
H(u, p) \geq \Phi(|z|) \quad \text { on } \mathbb{R}^{N} \times \mathbb{R}^{N},
$$

where $\Phi: \mathbb{R}^{+} \rightarrow \mathbb{R}^{+}$is a continuous function, strictly increasing and unbounded, and $z$ is a $j$-vector defined by (1.7).

REMARK 3.1. If there exists a real constant $\epsilon>0$ such that

$$
\tau^{\prime}(s) \geq \epsilon \quad \text { on } \mathbb{R}^{+},
$$

then, in view of (2.2), condition (3.1) holds with $\Phi(s)=h(\epsilon s)$ and $z=y$.

For the convenience of the reader, we recall the following classical definitions of boundedness [13, Ch. II] 
DEFINITION 3.1. A solution $u(t)=u\left(t, t_{0}, u_{0}, \dot{u}_{0}\right)$ of (1.1) is said to be $z$-bounded (in the future) if there exists a constant $C>0$ such that

$$
|z(t)| \leq C \quad \text { on }\left[t_{0}, \infty\right),
$$

where, of course, $z(t)=\left(\dot{u}_{k_{1}}(t), \ldots, \dot{u}_{k_{j}}(t)\right)$.

DEFINITION 3.2. The solutions of (1.1) are $z$-equi-bounded (in the future) if for any $B>0$ and $t_{0} \geq 0$, there exists a constant $C$, depending on $t_{0}$ and $B$, such that

$$
|z(t)| \leq C
$$

for any $t \geq t_{0}$ and $\left|u_{0}\right|+\left|\dot{u}_{0}\right| \leq B$.

DEFINITION 3.3. The solutions of (1.1) are $z$-uniform-bounded (in the future) if constant $C$ in Definition 3.2 does not depend on $t_{0}$.

Let $V=V(t, u, p)$ be the Liapunov function defined by (2.6).

ThEOREM 3.1. Suppose that conditions (2.1), (2.2), (2.3), and (3.1) hold. Moreover, assume that

(i) there exist three continuous functions $\mu, \rho: \mathbb{R}^{+} \rightarrow \mathbb{R}$ and $\phi: \mathbb{R}^{+} \rightarrow \mathbb{R}^{+}$such that

$$
\begin{aligned}
(Q(t, u, p), p)+F_{t}(t, u) \leq & \mu(t) \phi(V)+\rho(t) \psi(V) \\
& -2 \dot{\sigma}(t) \tau(|x|) f^{\prime}(\sigma(t) \tau(|x|)) \quad \text { on } \mathbb{R}^{+} \times \mathbb{R}^{N} \times \mathbb{R}^{N},
\end{aligned}
$$

(ii) $c(t)=\max _{t \in \mathbb{R}^{+}}\{\mu(t), \rho(t), 2 \sigma(t)\} \in L^{1}\left(\mathbb{R}^{+}\right)$,

(iii) $\int^{\infty} d s /\left(s+(\phi \circ f)(s) / f^{\prime}(s)+\left(h^{-1} \circ f\right)(s)\right)=\infty$.

Then all the solutions of (1.1) exist globally in the future and are z-equi-bounded.

Furthermore, if there exists a continuous function $V_{*}: \mathbb{R}^{N} \times \mathbb{R}^{N} \rightarrow \mathbb{R}^{+}$such that

(iv) $V(t, u, p) \leq V_{*}(u, p)$ on $\mathbb{R}^{+} \times \mathbb{R}^{N} \times \mathbb{R}^{N}$.

Then all the solutions of (1.1) are $z$-uniform-bounded.

Proof. Theorem 2.1 ensures that all the solutions of the system (1.1) exist globally in the future. To prove the $z$-equi-boundedness, consider two constants $B>0$ and $t_{0} \geq 0$, and put

$$
\tilde{v}_{0}=\max \left\{V\left(t_{0}, u_{0}, \dot{u}_{0}\right):\left|u_{0}\right|+\left|\dot{u}_{0}\right| \leq B\right\} .
$$

Let $u(t)=u\left(t, t_{0}, u_{0}, \dot{u}_{0}\right)$ be a solution of (1.1) with $\left|u_{0}\right|+\left|\dot{u}_{0}\right| \leq B$, and put $v(t)=$ $V(t, u(t), \dot{u}(t))$. Repeating the second part of the proof of Theorem 2.1, we obtain

$$
\dot{v} \leq c(t)\left[\phi(v)+\left(f^{\prime} \circ f^{-1}\right)(v) f^{-1}(v)+\left(f^{\prime} \circ f^{-1}\right)(v) h^{-1}(v)\right] \quad \text { on }\left[t_{0}, \infty\right),
$$

where

$$
c(t)=\max _{t \in \mathbb{R}^{+}}\{\mu(t), \rho(t), 2 \sigma(t)\} .
$$

Let us consider the comparison equation

$$
\dot{w}=c(t)\left[\phi(w)+\left(f^{\prime} \circ f^{-1}\right)(w) f^{-1}(w)+\left(f^{\prime} \circ f^{-1}\right)(w) h^{-1}(w)\right] .
$$


As before, we see that condition (iii) is equivalent to (2.25). Therefore, conditions (ii) and (iii) ensure that all the solutions of the comparison equation (3.9) are uniformbounded [7]. Thus, there exists a constant $w_{*}$, depending on $\tilde{v}_{0}$, such that

$$
w\left(t, t_{0}, \tilde{v}_{0}\right) \leq w_{*} \text { on }\left[t_{0}, \infty\right) .
$$

Since $v\left(t_{0}\right) \leq \tilde{v}_{0}$, by the comparison method, we have

$$
v(t) \leq w\left(t, t_{0}, \tilde{v}_{0}\right) \quad \text { on }\left[t_{0}, \infty\right) .
$$

Hence, from (3.10),

$$
v(t) \leq w_{*} \text { on }\left[t_{0}, \infty\right)
$$

Finally, (2.1) and (3.1) imply that

$$
\Phi(|z(t)|) \leq H(u(t), \dot{u}(t)) \leq v(t) \text { on }\left[t_{0}, \infty\right) .
$$

Thus, combining (3.12) and (3.13), we obtain

$$
|z(t)| \leq \Phi^{-1}\left(w_{*}\right) \quad \text { on }\left[t_{0}, \infty\right) \text {. }
$$

Since $w_{*}$ depends on $\tilde{v}_{0}$, and $\tilde{v}_{0}$ depends on $t_{0}$ and $B$, the solutions of (1.1) are $z$-equibounded.

Moreover, assume that condition (iv) holds too. Then if we repeat the proof with

$$
\tilde{v}_{0}=\max \left\{V_{*}\left(u_{0}, \dot{u}_{0}\right):\left|u_{0}\right|+\left|\dot{u}_{0}\right| \leq B\right\},
$$

we easily see that the constant $w_{*}$ does not depend on $t_{0}$. This completes the proof.

Theorem 3.1 has the following corollary, which substantially coincides with [5, Thm. 1'].

COROLLARY 3.1. Suppose that conditions (2.3) and (3.2) hold. Moreover, assume that

$$
\begin{gathered}
H(u, p) \geq\left(\tau^{\prime}(|x|)|y|\right)^{m} \quad \text { on } \mathbb{R}^{N} \times \mathbb{R}^{N}, \\
F(t, u) \geq-(\sigma(t) \tau(|x|))^{n} \quad \text { on } \mathbb{R}^{+} \times \mathbb{R}^{N}, \\
(Q(t, u, p), p)+F_{t}(t, u) \leq-2 n \frac{\dot{\sigma}(t)}{\sigma(t)}(\sigma(t) \tau(|x|))^{n} \\
+v(t)\left[\left(\tau^{\prime}(|x|)|y|\right)^{m}+(\sigma(t) \tau(|x|))^{n}\right] \quad \text { on } \mathbb{R}^{+} \times \mathbb{R}^{N} \times \mathbb{R}^{N},
\end{gathered}
$$

where $m \geq n \geq 1$ are real constants, $\sigma \in L^{1}\left(\mathbb{R}^{+}\right)$and $v: \mathbb{R}^{+} \rightarrow \mathbb{R}^{+}$is a continuous function of class $L^{1}\left(\mathbb{R}^{+}\right)$.

Then all the solutions of (1.1) exist globally in the future and are $y$-equi-bounded.

Furthermore, if $\sigma$ is bounded on $\mathbb{R}^{+}$and there exists a continuous function $F_{*}$ : $\mathbb{R}^{N} \rightarrow \mathbb{R}$ such that

$$
F(t, u) \leq F_{*}(u) \quad \text { on } \mathbb{R}^{+} \times \mathbb{R}^{N},
$$

then the solutions of (1.1) are $y$-uniform-bounded. 
Proof. Taking into account Remark 3.1, we easily verify that all the conditions of Theorem 3.1 are satisfied by choosing $f(s)=s^{n}, h(s)=s^{m}, \mu(t)=0, \rho(t)=v(t) / n$ and $z=y$.

REMARK 3.2. It is easy to prove that conditions (i), (ii), and (iii) of Theorem 3.1 can be replaced by the following

$$
\begin{gathered}
(Q(t, u, p), p)+F_{t}(t, u) \\
\leq \rho(t) \psi(V)-2 \dot{\sigma}(t) \tau(|x|) f^{\prime}(\sigma(t) \tau(|x|)) \quad \text { on } \mathbb{R}^{+} \times \mathbb{R}^{N} \times \mathbb{R}^{N}, \\
c(t)=\max _{t \in \mathbb{R}^{+}}\{\rho(t), 2 \sigma(t)\} \in L^{1}\left(\mathbb{R}^{+}\right), \\
\int^{\infty} \frac{d s}{s+\left(h^{-1} \circ f\right)(s)}=\infty,
\end{gathered}
$$

or

$$
\begin{gathered}
(Q(t, u, p), p)+F_{t}(t, u) \\
\leq-2 \dot{\sigma}(t) \tau(|x|) f^{\prime}(\sigma(t) \tau(|x|)) \quad \text { on } \mathbb{R}^{+} \times \mathbb{R}^{N} \times \mathbb{R}^{N}, \quad \sigma \in L^{1}\left(\mathbb{R}^{+}\right), \\
\quad \int^{\infty} \frac{d s}{\left(h^{-1} \circ f\right)(s)}=\infty .
\end{gathered}
$$

As in Section 2, from now on, we assume that

$$
\int^{\infty} \frac{d s}{\left(h^{-1} \circ f\right)(s)}<\infty
$$

and we consider two possibilities.

CASE 1. Assume that there exists a continuous function $\mu: \mathbb{R}^{+} \rightarrow \mathbb{R}$, with $\int^{\infty} \mu(s) d s$ $<\infty$, such that

$$
\begin{aligned}
& (Q(t, u, p), p)+F_{t}(t, u) \\
& \quad \leq \mu(t) V-2 \dot{\sigma}(t) \tau(|x|) f^{\prime}(\sigma(t) \tau(|x|)) \quad \text { on } \mathbb{R}^{+} \times \mathbb{R}^{N} \times \mathbb{R}^{N},
\end{aligned}
$$

and denote by $J(M)$ the range of the function $M(t)$, defined by (2.38).

Letting $t_{0} \geq 0$ and putting $M_{0}=M\left(t_{0}\right)$, the following result holds.

ThEOREM 3.2. Suppose that conditions (2.1), (2.2), (2.3), (3.1), and (3.26) hold. Moreover, assume that there exist two continuous functions $\alpha: \mathbb{R}^{+} \rightarrow \mathbb{R}^{+}$and $\beta: J(M) \rightarrow$ $(0, \infty)$, and a real constant $a_{0}>M_{0} f(0)$, such that

$$
\begin{gathered}
\left(f^{\prime} \circ f^{-1}\right)(s) h^{-1}(s) \leq \frac{\alpha(r s)}{\beta(r)} \quad \text { on } J(M) \times[f(0), \infty), \\
2 \int_{t_{0}}^{\infty} \frac{\sigma(t) M(t)}{\beta(M(t))} d t=\int_{a_{0}}^{\infty} \frac{d s}{\alpha(s)} .
\end{gathered}
$$

Then the solutions $u\left(t, t_{0}, u_{0}, \dot{u}_{0}\right)$ of (1.1) such that $V\left(t_{0}, u_{0}, \dot{u}_{0}\right)<a_{0} / M_{0}$ exist globally in the future and are $z$-bounded.

Proof. Let $u(t)=u\left(t, t_{0}, u_{0}, \dot{u}_{0}\right)$ be a solution of (1.1) with $v_{0}=V\left(t_{0}, u_{0}, \dot{u}_{0}\right)<$ $a_{0} / M_{0}$. Theorem 2.2 ensures that $u(t)$ exists globally in the future. Moreover, repeat- 
ing word-for-word the proof of Theorem 2.2, we obtain

$$
v(t) \leq \frac{w(t)}{M(t)} \text { on }\left[t_{0}, \infty\right),
$$

where $w(t)$ is the solution of the comparison equation (2.47) satisfying the initial condition $w\left(t_{0}\right)=M_{0} v_{0}<a_{0}$.

In view of (2.79), condition (3.28) implies that

$$
2 \int_{t_{0}}^{\infty} \frac{\sigma(t) M(t)}{\beta(M(t))} d t<\int_{M_{0} v_{0}}^{\infty} \frac{d s}{\alpha(s)},
$$

which ensures that the solution $w(t)$ is bounded [4], i.e., there exists a real constant $w_{*}$ such that $w(t) \leq w_{*}$ for every $t \geq t_{0}$.

Finally, as $\int^{\infty} \mu(s) d s<\infty$, there exists a real constant $M_{*}>0$ such that $M(t) \geq M_{*}$ for every $t \geq 0$. In view of (3.29), we, thus, have

$$
v(t) \leq \frac{w_{*}}{M_{*}} \text { on }\left[t_{0}, \infty\right),
$$

and the $z$-boundedness follows as before.

CASE 2. Assume that there exists a continuous function $\rho: \mathbb{R}^{+} \rightarrow \mathbb{R}$, with $\int^{\infty} \rho(s) d s$ $<\infty$, such that

$$
\begin{aligned}
& (Q(t, u, p), p)+F_{t}(t, u) \\
& \quad \leq \rho(t) \psi(V)-2 \dot{\sigma}(t) \tau(|x|) f^{\prime}(\sigma(t) \tau(|x|)) \quad \text { on } \mathbb{R}^{+} \times \mathbb{R}^{N} \times \mathbb{R}^{N},
\end{aligned}
$$

and denote by $J(P)$ the range of the function $P(t)$ defined by (2.52).

Let $t_{0} \geq 0$ be given, and $P_{0}=P\left(t_{0}\right)$. The following result holds.

Theorem 3.3. Suppose that conditions (2.1), (2.2), (2.3), (3.1), and (3.32) hold. Moreover, assume that there exist two continuous functions $\tilde{\alpha}: \mathbb{R}^{+} \rightarrow \mathbb{R}^{+}$and $\tilde{\beta}: J(P) \rightarrow$ $(0, \infty)$, and a real constant $b_{0}>0$, such that

$$
\begin{gathered}
\left(h^{-1} \circ f\right)(s) \leq \frac{\tilde{\alpha}(r s)}{\tilde{\beta}(r)} \quad \text { on } J(P) \times \mathbb{R}^{+}, \\
2 \int_{t_{0}}^{\infty} \frac{\sigma(t) P(t)}{\tilde{\beta}(P(t))} d t=\int_{b_{0}}^{\infty} \frac{d s}{\tilde{\alpha}(s)} .
\end{gathered}
$$

Then the solutions $u\left(t, t_{0}, u_{0}, \dot{u}_{0}\right)$ of (1.1) such that $V\left(t_{0}, u_{0}, \dot{u}_{0}\right)<f\left(b_{0} / P_{0}\right)$ exist globally in the future and are $z$-bounded.

Proof. Let $u(t)=u\left(t, t_{0}, u_{0}, \dot{u}_{0}\right)$ be a solution of (1.1) with $v_{0}=V\left(t_{0}, u_{0}, \dot{u}_{0}\right)<$ $f\left(b_{0} / P_{0}\right)$. Theorem 2.3 ensures that $u(t)$ exists globally in the future. Moreover, repeating word-for-word the proof of Theorem 2.3, we obtain

$$
v(t) \leq f\left(\frac{w(t)}{P(t)}\right) \quad \text { on }\left[t_{0}, \infty\right),
$$

where $w(t)$ is the solution of the comparison equation (2.61) satisfying the initial condition $w\left(t_{0}\right)=P_{0} f^{-1}\left(v_{0}\right)<b_{0}$. 
Consequently, by (2.80) and condition (3.34),

$$
2 \int_{t_{0}}^{\infty} \frac{\sigma(t) P(t)}{\tilde{\beta}(P(t))} d t<\int_{P_{0} f^{-1}\left(v_{0}\right)}^{\infty} \frac{d s}{\tilde{\alpha}(s)}
$$

which ensures that the solution $w(t)$ is bounded.

The rest of the proof follows exactly that of Theorem 3.2.

Theorems 3.2 and 3.3 have the following corollaries, whose proofs are the same as those of Corollaries 2.2 and 2.3, respectively.

COROLlary 3.2. Fix $t_{0} \geq 0$ and assume that $2 \int_{t_{0}}^{\infty} \sigma(t) d t<\int_{0}^{\infty} d s /\left(\left(h^{-1} \circ f\right)(s)\right)$. Moreover, suppose that (2.1), (2.2), (2.3), (3.1), and condition (2.63) of Corollary 2.2 hold. If $r_{0}>0$ is the real constant satisfying (2.64), then the solutions $u\left(t, t_{0}, u_{0}, \dot{u}_{0}\right)$ of (1.1) such that $V\left(t_{0}, u_{0}, \dot{u}_{0}\right)<f\left(r_{0}\right)$ exist globally in the future and are $z$-bounded.

COROLLARY 3.3. Suppose that all the conditions of Corollary 2.3 hold together with (3.2). Moreover, assume that $\int^{\infty} \rho(s) d s<\infty$.

Then the solutions $u\left(t, t_{0}, u_{0}, \dot{u}_{0}\right)$ of (1.1) such that

$$
H\left(u_{0}, \dot{u}_{0}\right)+F\left(t_{0}, u_{0}\right)+2\left(\sigma\left(t_{0}\right) \tau\left(\left|x_{0}\right|\right)\right)^{n}<P_{0}^{-n}\left[2 \lambda \int_{t_{0}}^{\infty} \sigma(t)(P(t))^{-\lambda} d t\right]^{-n / \lambda}
$$

exist globally in the future are $y$-bounded.

4. Global existence in the future, Part 2. In Sections 4 and 5, we assume the following

(a) $f: \mathbb{R}^{+} \rightarrow \mathbb{R}^{+}$is a function of class $C^{1}$, strictly increasing and unbounded, and such that $s f^{\prime}(s)$ is increasing;

(b) $g: \mathbb{R}^{+} \rightarrow \mathbb{R}^{+}$is a continuous function such that $g(s) f^{\prime}(s)$ is increasing;

(c) $\Gamma: \mathbb{R}^{+} \rightarrow \mathbb{R}^{+}$is a continuous function, with $\Gamma(0)=0$ and $\Gamma(s) / s \rightarrow \infty$ as $s \rightarrow \infty$;

(d) $\sigma: \mathbb{R}^{+} \rightarrow(0, \infty)$ is a function of class $C^{1}$;

(e) $a: \mathbb{R}^{+} \rightarrow(0, \infty)$ is a continuous function.

The main hypotheses of these sections follow.

$$
\begin{gathered}
F(t, u) \geq-f(\sigma(t)|x|) \quad \text { on } \mathbb{R}^{+} \times \mathbb{R}^{N}, \\
(Q(t, u, p), p)+F_{t}(t, u) \leq \mu(t) \phi(V)+\rho(t) \psi(V) \\
-a(t) f^{\prime}(\sigma(t)|x|) g(\sigma(t)|x|) \Gamma\left(\frac{|y|}{g(\sigma(t)|x|)}\right) \quad \text { on } \mathbb{R}^{+} \times \mathbb{R}^{N} \times \mathbb{R}^{N},
\end{gathered}
$$

where $\mu, \rho: \mathbb{R}^{+} \rightarrow \mathbb{R}$ and $\phi: \mathbb{R}^{+} \rightarrow \mathbb{R}^{+}$are continuous functions, and

$$
V(t, u, p)=H(u, p)+F(t, u)+2 f(\sigma(t)|x|)
$$

is the new Liapunov function.

Moreover, as in Sections 2 and 3, we assume that, corresponding to each constant $\omega>0$, there exists a continuous, strictly increasing and unbounded function $\Phi_{\omega}$ such that

$$
H(u, p)>\Phi_{\omega}(|p|) \quad \text { on } \Omega \times \mathbb{R}^{N} .
$$


REMARK 4.1. To understand the meaning of condition (4.2), let us consider the particular case when $F_{t}(t, u) \geq 0, \mu(t) \leq 0$, and $\rho(t) \leq 0$. Then (4.2) implies

$$
(Q(t, u, p), p) \leq-a(t) D(t, x, y) \quad \text { on } \mathbb{R}^{+} \times \mathbb{R}^{N} \times \mathbb{R}^{N},
$$

where, for simplicity, we write

$$
D(t, x, y)=f^{\prime}(\sigma(t)|x|) g(\sigma(t)|x|) \Gamma\left(\frac{|y|}{g(\sigma(t)|x|)}\right) .
$$

We observe that

$$
D(t, x, y) \geq 0 \quad \text { on } \mathbb{R}^{+} \times \mathbb{R}^{i} \times \mathbb{R}^{i} \quad \text { and } \quad D(t, x, 0)=0 \quad \text { on } \mathbb{R}^{+} \times \mathbb{R}^{i} .
$$

Therefore, recalling that (1.1) may be considered as the motion law of a holonomic dynamical system, the vector $Q$ represents a continuous damping term.

Furthermore, let $k, n, m$ be real constants such that $k>1, n \geq 1$, and $1-n \leq m \leq$ $(n-1) /(k-1)$. Then if

$$
f(s)=s^{n}, \quad g(s)=s^{m}, \quad \Gamma(s)=s^{k},
$$

condition (4.5) becomes

$$
(Q(t, u, p), p) \leq-b(t)|x|^{h}|y|^{k} \quad \text { on } \mathbb{R}^{+} \times \mathbb{R}^{N} \times \mathbb{R}^{N},
$$

where $h=n-1+m-k m$ is a positive constant, and $b(t)=n a(t)(\sigma(t))^{h}$.

A condition of the type (4.9) has been considered by the author in [5, Thms. 2, 2'], [2, Thm. 4], and [1], and by Pucci and Serrin in [10, Thms. 3.2, 4.1].

THEOREM 4.1. Suppose that (4.1) and (4.4) hold. Moreover, assume that

$$
\begin{gathered}
(Q(t, u, p), p)+F_{t}(t, u) \\
\leq \mu(t) \phi(V)+\rho(t) \psi(V)-a(t) D(t, x, y) \quad \text { on } \mathbb{R}^{+} \times \mathbb{R}^{N} \times \mathbb{R}^{N}, \\
\quad \int^{\infty} \frac{d s}{s+(\phi \circ f)(s) / f^{\prime}(s)+g(s)}=\infty .
\end{gathered}
$$

Then all solutions of (1.1) exist globally in the future.

Before giving the proof, it is worth recalling that $\Gamma(s) / s \rightarrow \infty$ as $s \rightarrow \infty$. This ensures that, corresponding to the given functions $a(t)$ and $\sigma(t)$, there exists a continuous function $\delta: \mathbb{R}^{+} \rightarrow \mathbb{R}^{+}$such that

$$
s-\frac{a(t)}{2 \sigma(t)} \Gamma(s) \geq \frac{\delta(t)}{2 \sigma(t)} \quad \text { for each } t \geq 0 \text { and } s \geq 0 .
$$

For example, if $\Gamma(s)=s^{k}$ with $k>1$, the best function $\delta$ satisfying (4.12) is given by

$$
\delta(t)=2 \frac{k-1}{k} \sigma(t)\left[\frac{2 \sigma(t)}{k a(t)}\right]^{1 /(k-1)} .
$$

We leave these verifications to the reader. 
Proof OF THEOREM 4.1. Suppose for contradiction that there exists a solution $u(t)$ of (1.1) defined on a right maximal interval of existence $\left[t_{0}, T\right)$, with $t_{0}<T<\infty$. Then (2.7) holds.

Moreover, put $v(t)=V(t, u(t), \dot{u}(t))$, where $V(t, u, p)$ is given by (4.3). Then, following nearly word-by-word the first part of the proof of Theorem 2.1, we can show that

$$
\limsup _{t \rightarrow T^{-}} v(t)=\infty .
$$

Let us consider the derivative of function $v(t)$

$$
\begin{aligned}
\dot{v}=(Q(t, u, p), p)+F_{t}(t, u)+ & 2 f^{\prime}(\sigma(t)|x|) \\
& \times\left[\dot{\sigma}(t)|x|+\sigma(t) \frac{d|x|}{d t}\right] \quad \text { on }\left[t_{0}, T\right) .
\end{aligned}
$$

By condition (4.10) and the fact that $(d / d t)|x(t)| \leq|\dot{x}(t)|$, we, then, obtain, for each $t \in\left[t_{0}, T\right)$,

$$
\begin{aligned}
\dot{v} \leq & \mu(t) \phi(v)+\rho(t) \psi(v)-a(t) f^{\prime}(\sigma(t)|x|) g(\sigma(t)|x|) \Gamma\left(\frac{|\dot{x}|}{g(\sigma(t)|x|)}\right) \\
& +2 \dot{\sigma}(t)|x| f^{\prime}(\sigma(t)|x|)+2 \sigma(t)|\dot{x}| f^{\prime}(\sigma(t)|x|) \\
= & \mu(t) \phi(v)+\rho(t) \psi(v)+2 \dot{\sigma}(t)|x| f^{\prime}(\sigma(t)|x|) \\
& +2 \sigma(t) f^{\prime}(\sigma(t)|x|) g(\sigma(t)|x|)\left[\frac{|\dot{x}|}{g(\sigma(t)|x|)}-\frac{a(t)}{2 \sigma(t)} \Gamma\left(\frac{|\dot{x}|}{g(\sigma(t)|x|)}\right)\right] .
\end{aligned}
$$

If in (4.12) we put $s=|\dot{x}| / g(\sigma(t)|x|)$, we see that there exists a continuous function $\delta: \mathbb{R}^{+} \rightarrow \mathbb{R}^{+}$such that

$$
\frac{|\dot{x}|}{g(\sigma(t)|x|)}-\frac{a(t)}{2 \sigma(t)} \Gamma\left(\frac{|\dot{x}|}{g(\sigma(t)|x|)}\right) \leq \frac{\delta(t)}{2 \sigma(t)} \quad \text { on }\left[t_{0}, T\right) .
$$

Thus, from (4.16), we obtain

$$
\begin{gathered}
\dot{v} \leq \mu(t) \phi(v)+\rho(t) \psi(v)+2 \dot{\sigma}(t)|x| f^{\prime}(\sigma(t)|x|) \\
+\delta(t) f^{\prime}(\sigma(t)|x|) g(\sigma(t)|x|) \text { on }\left[t_{0}, T\right) .
\end{gathered}
$$

By virtue of (4.1), we have

$$
\sigma(t)|x(t)| \leq f^{-1}(v(t)) \text { on }\left[t_{0}, T\right) .
$$

So, we have, since $s f^{\prime}(s)$ and $f^{\prime}(s) g(s)$ are increasing functions,

$$
\begin{aligned}
\sigma(t)|x| f^{\prime}(\sigma(t)|x|) & \leq\left(f^{\prime} \circ f^{-1}\right)(v(t)) f^{-1}(v(t)) \quad \text { on }\left[t_{0}, T\right), \\
f^{\prime}(\sigma(t)|x|) g(\sigma(t)|x|) & \leq\left(f^{\prime} \circ f^{-1}\right)(v(t))\left(g \circ f^{-1}\right)(v(t)) \quad \text { on }\left[t_{0}, T\right) .
\end{aligned}
$$

Hence, putting

$$
c(t)=\max _{t \in \mathbb{R}^{+}}\left\{\mu(t), \rho(t)+2 \frac{|\dot{\sigma}(t)|}{\sigma(t)}, \delta(t)\right\}
$$


gives

$$
\begin{aligned}
\dot{v} \leq c(t)[\phi(v) & +\left(f^{\prime} \circ f^{-1}\right)(v) f^{-1}(v) \\
& \left.+\left(f^{\prime} \circ f^{-1}\right)(v)\left(g \circ f^{-1}\right)(v)\right] \quad \text { on }\left[t_{0}, T\right) .
\end{aligned}
$$

Consider the comparison equation

$$
\dot{w}=c(t)\left[\phi(w)+\left(f^{\prime} \circ f^{-1}\right)(w) f^{-1}(w)+\left(f^{\prime} \circ f^{-1}\right)(w)\left(g \circ f^{-1}\right)(w)\right] .
$$

Let $a, b$ be real positive constants. Since

$$
\begin{aligned}
& \int_{a}^{b} \frac{d s}{s+(\phi \circ f)(s) / f^{\prime}(s)+g(s)} \\
& \quad=\int_{f(a)}^{f(b)} \frac{d s}{\phi(s)+\left(f^{\prime} \circ f^{-1}\right)(s) f^{-1}(s)+\left(f^{\prime} \circ f^{-1}\right)(s)\left(g \circ f^{-1}\right)(s)},
\end{aligned}
$$

and since $f(s) \rightarrow \infty$ as $s \rightarrow \infty$, by condition (4.11), it follows that

$$
\int^{\infty} \frac{d s}{\phi(s)+\left(f^{\prime} \circ f^{-1}\right)(s) f^{-1}(s)+\left(f^{\prime} \circ f^{-1}\right)(s)\left(g \circ f^{-1}\right)(s)}=\infty .
$$

Then all the solutions of the comparison equation (4.23) exist globally in the future [7].

Denote by $w(t)$ a solution of (4.23) satisfying the initial condition $w\left(t_{0}\right) \geq v\left(t_{0}\right)=$ $V\left(t_{0}, u_{0}, \dot{u}_{0}\right)$. By comparison, it follows that

$$
v(t) \leq w(t) \text { on }\left[t_{0}, T\right),
$$

which contradicts (4.14) since $w(t)$ is a continuous function defined on $\left[t_{0}, \infty\right)$. This contradiction completes the proof.

Theorem 4.1 has the following corollary, coinciding with [5, Thm. 2].

COROLlary 4.1. Suppose that condition (4.4) holds. Moreover, assume that there exist three real constants $h \geq 0, k>1$ and $n \geq 1+h / k$ such that

$$
\begin{gathered}
F(t, u) \geq-(\sigma(t)|x|)^{n} \quad \text { on } \mathbb{R}^{+} \times \mathbb{R}^{N}, \\
(Q(t, u, p), p)+F_{t}(t, u) \\
\leq v(t)\left[H(u, p)+(\sigma(t)|x|)^{n}\right]-b(t)|x|^{h}|y|^{k} \quad \text { on } \mathbb{R}^{+} \times \mathbb{R}^{N} \times \mathbb{R}^{N},
\end{gathered}
$$

where $v: \mathbb{R}^{+} \rightarrow \mathbb{R}^{+}$and $b: \mathbb{R}^{+} \rightarrow(0, \infty)$ are continuous functions.

Then all solutions of (1.1) exist globally in the future if $n \leq h+k$.

Proof. We see that if

$$
\begin{aligned}
& f(s)=s^{n}, \quad g(s)=s^{m}\left(m=\frac{n-h-1}{k-1}\right), \quad \Gamma(s)=s^{k}, \\
& a(t)=\frac{1}{n} b(t)(\sigma(t))^{-h}, \quad \mu(t)=0, \quad \rho(t)=\frac{v(t)}{n},
\end{aligned}
$$

then all the conditions of Theorem 4.1 are satisfied. 
REMARK 4.2. It is easy to verify that conditions (4.10) and (4.11) of Theorem 4.1 can be replaced by the following

$$
\begin{gathered}
(Q(t, u, p), p)+F_{t}(t, u) \leq \rho(t) \psi(V)-a(t) D(t, x, y) \quad \text { on } \mathbb{R}^{+} \times \mathbb{R}^{N} \times \mathbb{R}^{N}, \\
\int^{\infty} \frac{d s}{s+g(s)}=\infty .
\end{gathered}
$$

Or, alternately,

$$
\begin{aligned}
& (Q(t, u, p), p)+F_{t}(t, u) \\
& \leq-2 \dot{\sigma}(t)|x| f^{\prime}(\sigma(t)|x|)-a(t) D(t, x, y) \text { on } \mathbb{R}^{+} \times \mathbb{R}^{N} \times \mathbb{R}^{N}, \\
& \qquad \int^{\infty} \frac{d s}{g(s)}=\infty .
\end{aligned}
$$

In the remainder of the section, we assume that

$$
\int^{\infty} \frac{d s}{g(s)}<\infty
$$

Thus, we are unable to ensure global existence in the future of all the solutions of system (1.1). We consider two cases where there are submanifolds of solutions which exist globally in the future.

CASE 1. Assume that there exists a continuous function $\mu: \mathbb{R}^{+} \rightarrow \mathbb{R}$ such that

$$
\begin{aligned}
& (Q(t, u, p), p)+F_{t}(t, u) \\
& \quad \leq \mu(t) V-2 \dot{\sigma}(t)|x| f^{\prime}(\sigma(t)|x|)-a(t) D(t, x, y) \text { on } \mathbb{R}^{+} \times \mathbb{R}^{N} \times \mathbb{R}^{N},
\end{aligned}
$$

and denote by $J(M) \subset \mathbb{R}^{+}$the range of the function $M(t)=\exp \left\{-\int_{0}^{t} \mu(s) d s\right\}$. Moreover, let $\delta$ be a function satisfying (4.12), $t_{0} \geq 0$ and $M_{0}=M\left(t_{0}\right)$.

THEOREM 4.2. Suppose that conditions (4.1), (4.4), and (4.35) hold. Moreover, assume that there exist two continuous functions $\alpha: \mathbb{R}^{+} \rightarrow \mathbb{R}^{+}$and $\beta: J(M) \rightarrow(0, \infty)$, and a real constant $a_{0} \geq M_{0} f(0)$, such that

$$
\begin{gathered}
\left(f^{\prime} \circ f^{-1}\right)(s)\left(g \circ f^{-1}\right)(s) \leq \frac{\alpha(r s)}{\beta(r)} \text { on } J(M) \times[f(0), \infty), \\
\int_{t_{0}}^{\infty} \frac{\delta(t) M(t)}{\beta(M(t))} d t=\int_{a_{0}}^{\infty} \frac{d s}{\alpha(s)} .
\end{gathered}
$$

Then the solutions $u\left(t, t_{0}, u_{0}, \dot{u}_{0}\right)$ of (1.1) such that $V\left(t_{0}, u_{0}, \dot{u}_{0}\right) \leq a_{0} / M_{0}$ exist globally in the future.

Proof. The proof is parallel to that of Theorem 2.2. Let $u(t)=u\left(t, t_{0}, u_{0}, \dot{u}_{0}\right)$ be a solution of (1.1) and $v_{0}=V\left(t_{0}, u_{0}, \dot{u}_{0}\right) \leq a_{0} / M_{0}$. Assume for contradiction that $\left[t_{0}, T\right)$ is maximal for $u(t)$, and put $v(t)=V(t, u(t), \dot{u}(t))$.

Repeating the proof of Theorem 4.1 with $\phi(s)=s$, we obtain

$$
\dot{v} \leq \mu(t) v+\delta(t)\left(f^{\prime} \circ f^{-1}\right)(v)\left(g \circ f^{-1}\right)(v) \text { on }\left[t_{0}, T\right) .
$$


Then, putting $q(t)=M(t) v(t)$, we see that

$$
\dot{q} \leq \delta(t) M(t)\left(f^{\prime} \circ f^{-1}\right)(v)\left(g \circ f^{-1}\right)(v) \quad \text { on }\left[t_{0}, T\right),
$$

from which follows, in view of condition (4.36),

$$
\dot{q} \leq \frac{\delta(t) M(t)}{\beta(M(t))} \alpha(q) \quad \text { on }\left[t_{0}, T\right) .
$$

Since $q\left(t_{0}\right)=M_{0} v_{0} \leq a_{0}$, condition (4.37) ensures that the solution $w(t)=w\left(t, t_{0}\right.$, $\left.q\left(t_{0}\right)\right)$ of the comparison equation

$$
\dot{w}=\frac{\delta(t) M(t)}{\beta(M(t))} \alpha(w)
$$

exists globally in the future.

Finally, by comparison, we get

$$
M(t) v(t)=q(t) \leq w(t) \quad \text { on }\left[t_{0}, T\right),
$$

which contradicts (4.14). This completes the proof.

CASE 2. Assume that there exists a continuous function $\rho: \mathbb{R}^{+} \rightarrow \mathbb{R}$ such that

$$
\begin{aligned}
& (Q(t, u, p), p)+F_{t}(t, u) \\
& \quad \leq \rho(t) \psi(t)-2 \dot{\sigma}(t)|x| f^{\prime}(\sigma(t)|x|)-a(t) D(t, x, y) \quad \text { on } \mathbb{R}^{+} \times \mathbb{R}^{N} \times \mathbb{R}^{N},
\end{aligned}
$$

and denote by $J(P) \subset \mathbb{R}^{+}$the range of the function $P(t)=\exp \left\{-\int_{0}^{t} \rho(s) d s\right\}$. Moreover, let $\delta$ be a function satisfying (4.12), $t_{0} \geq 0$ and $M_{0}=M\left(t_{0}\right)$.

THEOREM 4.3. Suppose that conditions (4.1), (4.4), and (4.43) hold. Moreover, assume that there exist two continuous functions $\tilde{\alpha}: \mathbb{R}^{+} \rightarrow \mathbb{R}^{+}$and $\tilde{\beta}: J(P) \rightarrow(0, \infty)$, and a real constant $b_{0} \geq 0$, such that

$$
\begin{gathered}
g(s) \leq \frac{\tilde{\alpha}(r s)}{\tilde{\beta}(r)} \quad \text { on } J(P) \times \mathbb{R}^{+}, \\
\int_{t_{0}}^{\infty} \frac{\delta(t) P(t)}{\tilde{\beta}(P(t))} d t=\int_{b_{0}}^{\infty} \frac{d s}{\tilde{\alpha}(s)} .
\end{gathered}
$$

Then the solutions $u\left(t, t_{0}, u_{0}, \dot{u}_{0}\right)$ of (1.1) such that $V\left(t_{0}, u_{0}, \dot{u}_{0}\right) \leq f\left(b_{0} / P_{0}\right)$ exist globally in the future.

Proof. The proof is parallel to that of Theorem 2.3. Let $u(t)=u\left(t, t_{0}, u_{0}, \dot{u}_{0}\right)$ be a solution of (1.1) and $v_{0}=V\left(t_{0}, u_{0}, \dot{u}_{0}\right) \leq f\left(b_{0} / P_{0}\right)$. Assume for contradiction that $\left[t_{0}, T\right)$ is maximal for $u(t)$, and put $v(t)=V(t, u(t), \dot{u}(t))$.

The proof of Theorem 4.1 gives, by replacing condition (4.10) of Theorem 4.1 with (4.43),

$$
\dot{v} \leq\left(f^{\prime} \circ f^{-1}\right)(v)\left[\rho(t) f^{-1}(v)+\delta(t)\left(g \circ f^{-1}\right)(v)\right] \quad \text { on }\left[t_{0}, T\right) \text {. }
$$


Then, putting $q(t)=P(t) f^{-1}(v(t))$, we have

$$
\dot{q} \leq \delta(t) P(t)\left(g \circ f^{-1}\right)(v) \quad \text { on }\left[t_{0}, T\right),
$$

from which follows, in view of condition (4.44),

$$
\dot{q} \leq \frac{\delta(t) P(t)}{\tilde{\beta}(P(t))} \tilde{\alpha}(q) \quad \text { on }\left[t_{0}, T\right) .
$$

On the other hand, $q\left(t_{0}\right)=P_{0} f^{-1}\left(v_{0}\right) \leq b_{0}$. Thus, condition (4.45) ensures that the solution $w(t)=w\left(t, t_{0}, q\left(t_{0}\right)\right)$ of the comparison equation

$$
\dot{w}=\frac{\delta(t) P(t)}{\tilde{\beta}(P(t))} \tilde{\alpha}(w)
$$

exists globally in the future.

Finally, by comparison, we see that

$$
P(t) f^{-1}(v(t))=q(t) \leq w(t) \text { on }\left[t_{0}, T\right),
$$

which contradicts (4.14). This completes the proof.

Theorems 4.2 and 4.3 have the following corollaries. We omit their proofs since they are essentially the same as those of Corollaries 2.2 and 2.3, respectively.

COROLLARY 4.2. Fix $t_{0} \geq 0$ and assume that there is a function $\delta(t)$ satisfying (4.12) such that $\int_{0}^{\infty} \delta(t) d t \leq \int_{0}^{\infty} d s / g(s)$. Moreover, suppose that conditions (4.1) and (4.4) hold, together with

$$
\begin{aligned}
(Q(t, u, p), p) & +F_{t}(t, u) \\
\leq & -2 \dot{\sigma}(t)|x| f^{\prime}(\sigma(t)|x|)-a(t) D(t, x, y) \quad \text { on } \mathbb{R}^{+} \times \mathbb{R}^{N} \times \mathbb{R}^{N} .
\end{aligned}
$$

If $r_{0} \geq 0$ is the real constant satisfying

$$
\int_{t_{0}}^{\infty} \delta(t) d t=\int_{r_{0}}^{\infty} \frac{d s}{g(s)}
$$

then the solutions $u\left(t, t_{0}, u_{0}, \dot{u}_{0}\right)$ of (1.1) such that $V\left(t_{0}, u_{0}, \dot{u}_{0}\right) \leq f\left(r_{0}\right)$ exist globally in the future.

COROLlary 4.3. Let $h \geq 0, k>1$ and $n>h+k$ be real constants. Suppose that condition (4.4) holds, together with the following

$$
\begin{gathered}
F(t, u) \geq-(\sigma(t)|x|)^{n} \quad \text { on } \mathbb{R}^{+} \times \mathbb{R}^{N}, \\
(Q(t, u, p), p)+F_{t}(t, u) \\
\leq n \rho(t)\left[H(u, p)+(\sigma(t)|x|)^{n}\right]-2 n \frac{\dot{\sigma}(t)}{\sigma(t)}(\sigma(t)|x|)^{n} \\
-b(t)|x|^{h}|y|^{k} \quad \text { on } \mathbb{R}^{+} \times \mathbb{R}^{N} \times \mathbb{R}^{N},
\end{gathered}
$$

where $\rho: \mathbb{R}^{+} \rightarrow \mathbb{R}^{+}$and $b: \mathbb{R}^{+} \rightarrow(0, \infty)$ are continuous functions.

Moreover, putting $m=(n-h-1) /(k-1), P(t)=\exp \left\{-\int_{0}^{t} \rho(s) d s\right\}$ and

$$
\delta(t)=2 \frac{k-1}{k}\left[\frac{2 n}{k} \frac{(\sigma(t))^{h+k}}{b(t)}\right]^{1 /(k-1)}
$$


assume $\delta P^{1-m} \in L^{1}\left(\mathbb{R}^{+}\right)$.

Then the solutions $u\left(t, t_{0}, u_{o}, \dot{u}_{o}\right)$ of (1.1) such that

$$
\begin{aligned}
H\left(u_{0}, \dot{u}_{0}\right)+F\left(t_{0}, u_{0}\right) & +2\left(\sigma\left(t_{0}\right)\left|x_{0}\right|\right)^{n} \\
& \leq P_{0}^{-n}\left[(m-1) \int_{t_{0}}^{\infty} \delta(t)(p(t))^{1-m} d t\right]^{n /(1-m)}
\end{aligned}
$$

exist globally in the future.

REMARK 4.3. As regards to the proof of Corollary 4.2, we have to put $f(s)=s^{n}$, $\Gamma(s)=s^{k}$, and $g(s)=s^{m}$ in Theorem 4.2 (or in Theorem 4.3), and take into account (4.13).

5. Boundedness, Part 2. As in Section 3, we assume that there exists a continuous function $\Phi: \mathbb{R}^{+} \rightarrow \mathbb{R}^{+}$, strictly increasing and unbounded, such that

$$
H(u, p) \geq \Phi(|z|) \quad \text { on } \mathbb{R}^{N} \times \mathbb{R}^{N} .
$$

Denoting by $V(t, u, p)$ the Liapunov function defined by (4.3), the following result holds.

THEOREM 5.1. Suppose that conditions (4.1), (4.4) and (5.1) hold. Moreover, assume that there exist a continuous function $\delta: \mathbb{R}^{+} \rightarrow \mathbb{R}^{+}$satisfying (4.12), and three continuous functions $\mu, \rho: \mathbb{R}^{+} \rightarrow \mathbb{R}$ and $\phi: \mathbb{R}^{+} \rightarrow \mathbb{R}^{+}$, such that

(i) $c(t)=\max _{t \in \mathbb{R}^{+}}\{\mu(t), \rho(t), \delta(t)\} \in L^{1}\left(\mathbb{R}^{+}\right)$,

(ii) $(Q(t, u, p), p)+F_{t}(t, u) \leq \mu(t) \phi(V)+\rho(t) \psi(V)-2 \dot{\sigma}(t)|x| f^{\prime}(\sigma(t)|x|)$ $-a(t) D(t, x, y)$ on $\mathbb{R}^{+} \times \mathbb{R}^{N} \times \mathbb{R}^{N}$,

(iii) $\int^{\infty} d s /\left(s+(\phi \circ f)(s) / f^{\prime}(s)+g(s)\right)=\infty$.

Then all solutions of (1.1) exist globally in the future and are z-equi-bounded. Moreover, if (5.1) is replaced by

(iv) there exists a constant $\gamma>0$ such that $\sigma(t) \geq \gamma$ on $\mathbb{R}^{+}$.

Then all the solutions of (1.1) exist globally in the future and are $(x, p)$-equibounded.

Finally, if there is a continuous function $V_{*}: \mathbb{R}^{N} \times \mathbb{R}^{N} \rightarrow \mathbb{R}^{+}$such that

(v) $V(t, u, p) \leq V_{*}(u, p)$ on $\mathbb{R}^{+} \times \mathbb{R}^{N} \times \mathbb{R}^{N}$.

Then the above-mentioned type of boundedness are uniform.

Proof. By Theorem 4.1, it is enough to prove that the solutions of (1.1) are equi(or uniform-) bounded.

Fix $B>0$ and $t_{0} \geq 0$ and put

$$
\tilde{v}_{0}=\max \left\{V\left(t_{0}, u_{0}, \dot{u}_{0}\right):\left|u_{0}\right|+\left|\dot{u}_{0}\right| \leq B\right\} .
$$

Moreover, let $u(t)=u\left(t, t_{0}, u_{0}, \dot{u}_{0}\right)$ be a solution of (1.1) with $\left|u_{0}\right|+\left|\dot{u}_{0}\right| \leq B$, and put $v(t)=V(t, u(t), \dot{u}(t))$. Arguing as in the proof of Theorem 4.1, we can show that

$$
\begin{aligned}
\dot{v} \leq c(t)[\phi(v) & +\left(f^{\prime} \circ f^{-1}\right)(v) f^{-1}(v) \\
& \left.+\left(f^{\prime} \circ f^{-1}\right)(v)\left(g \circ f^{-1}\right)(v)\right] \text { on }\left[t_{0}, \infty\right),
\end{aligned}
$$


where

$$
c(t)=\max _{t \in \mathbb{R}^{+}}\{\mu(t), \rho(t), \delta(t)\} .
$$

Consider the comparison equation

$$
\dot{w}=c(t)\left[\phi(w)+\left(f^{\prime} \circ f^{-1}\right)(w) f^{-1}(w)+\left(f^{\prime} \circ f^{-1}\right)(w)\left(g \circ f^{-1}\right)(w)\right] .
$$

Since condition (iii) is equivalent to (4.25), conditions (i) and (iii) ensure that all the solutions of the comparison equation (5.5) are uniform-bounded. So, by the comparison method and the fact that $v\left(t_{0}\right) \leq \tilde{v}_{0}$, it follows that

$$
v(t) \leq w\left(t, t_{0}, \tilde{v}_{0}\right) \leq w_{*} \text { on }\left[t_{0}, \infty\right),
$$

where $w_{*}$ is a suitable constant which depends only on $\tilde{v}_{0}$.

Now, repeating the proof of Theorem 3.1, we easily see that the solutions of (1.1) are $z$-equi-bounded (or $z$-uniform-bounded if also condition (v) holds).

If condition (iv) replaces (5.1), then by (4.1) and (5.6), we obtain

$$
f(\sigma(t)|x|) \leq v(t) \leq w_{*} \text { on }\left[t_{0}, \infty\right),
$$

and, consequently,

$$
|x(t)| \leq \frac{f^{-1}\left(w_{*}\right)}{\gamma} \quad \text { on }\left[t_{0}, \infty\right)
$$

Put $\omega=f^{-1}\left(w_{*}\right) / \gamma$. Inequality (5.8) implies that $u(t) \in \Omega$ for each $t \geq t_{0}$. Then, by combining (4.4) and (5.6), we have

$$
\Phi_{\omega}(|\dot{u}(t)|) \leq v(t) \leq w_{*} \text { on }\left[t_{0}, \infty\right),
$$

i.e.,

$$
|\dot{u}(t)| \leq \Phi_{\omega}^{-1}\left(w_{*}\right) \quad \text { on }\left[t_{0}, \infty\right) .
$$

Inequalities (5.8) and (5.10) show that all the solutions of (1.1) are $(x, p)$-equibounded (note that $w_{*}$ depends on $B$ and $t_{0}$ ).

Finally, if condition (v) holds as well, then we can choose a constant $w_{*}$ which does not depend on $t_{0}$. This completes the proof.

Let $h \geq 0$ and $k>1$ be real constants. The following corollary of Theorem 5.1 holds.

COROLLARY 5.1. Suppose that the condition (4.4) holds. Moreover, assume that there exist $a$ constant $\gamma>0$ and a continuous function $b: \mathbb{R}^{+} \rightarrow(0, \infty)$ such that

$$
\begin{gathered}
F(t, u) \geq-(\sigma(t)|x|)^{n} \quad \text { on } \mathbb{R}^{+} \times \mathbb{R}^{N} ; \\
(Q(t, u, p), p)+F_{t}(t, u) \\
\leq-2 n \frac{\dot{\sigma}(t)}{\sigma(t)}(\sigma(t)|x|)^{n}-b(t)|x|^{h}|y|^{k} \quad \text { on } \mathbb{R}^{+} \times \mathbb{R}^{N} \times \mathbb{R}^{N} ; \\
\int^{\infty}\left[\frac{(\sigma(t))^{h+k}}{b(t)}\right]^{1 /(k-1)} d t<\infty \quad \text { and } \sigma(t) \geq \gamma \quad \text { on } \mathbb{R}^{+} .
\end{gathered}
$$


Then all the solutions of (1.1) exist globally in the future and are $(x, p)$-equi-bounded if $n \leq h+k$.

Proof. We can see that conditions (i)-(iv) of Theorem 5.1 are satisfied for $f(s)=$ $s^{n}, g(s)=s^{m}(m=(n-h-1) /(k-1)), \Gamma(s)=s^{k}, \mu(t)=\rho(t)=0$, and $a(t)=$ $b(t) /\left(n \sigma^{h}(t)\right)$. Here, because of (4.13), we have

$$
c(t)=\delta(t)=2 \frac{k-1}{k}\left[\frac{2 n}{k} \frac{(\sigma(t))^{h+k}}{b(t)}\right]^{1 /(k-1)} .
$$

REMARK 5.1. We can show without difficulty that conditions (i), (ii), and (iii) of Theorem 5.1 can be replaced by the following

$$
c(t)=\max _{t \in \mathbb{R}^{+}}\{\rho(t), \delta(t)\} \in L^{1}\left(\mathbb{R}^{+}\right),
$$

$$
\begin{aligned}
& (Q(t, u, p), p)+F_{t}(t, u) \\
& \leq \rho(t) \psi(V)-2 \dot{\sigma}(t)|x| f^{\prime}(\sigma(t)|x|)-a(t) D(t, x, y) \text { on } \mathbb{R}^{+} \times \mathbb{R}^{N} \times \mathbb{R}^{N}, \\
& \quad \int^{\infty} \frac{d s}{s+g(s)}=\infty ;
\end{aligned}
$$

or, alternately,

$$
\delta \in L^{1}\left(\mathbb{R}^{+}\right),
$$

$$
\begin{aligned}
& (Q(t, u, p), p)+F_{t}(t, u) \\
& \leq-2 \dot{\sigma}(t)|x| f^{\prime}(\sigma(t)|x|)-a(t) D(t, x, y) \text { on } \mathbb{R}^{+} \times \mathbb{R}^{N} \times \mathbb{R}^{N}, \\
& \qquad \int^{\infty} \frac{d s}{g(s)}=\infty .
\end{aligned}
$$

From here on, we assume that

$$
\int^{\infty} \frac{d s}{g(s)}<\infty
$$

a condition which, of course, makes Theorem 5.1 unusable.

We consider two cases.

CASE 1. Assume that there exists a continuous function $\mu: \mathbb{R}^{+} \rightarrow \mathbb{R}$, with $\int^{\infty} \mu(s) d s<$ $\infty$, such that

$$
\begin{aligned}
& (Q(t, u, p), p)+F_{t}(t, u) \\
& \quad \leq \mu(t) V-2 \dot{\sigma}(t)|x| f^{\prime}(\sigma(t)|x|)-a(t) D(t, x, y) \text { on } \mathbb{R}^{+} \times \mathbb{R}^{N} \times \mathbb{R}^{N},
\end{aligned}
$$

and denote by $J(M)$ the range of the function $M(t)$ defined by (2.38).

Fix $t_{0} \geq 0$ and put $M_{0}=M\left(t_{0}\right)$.

Theorem 5.2. Suppose that conditions (4.1), (4.4), (5.1), and (5.22) hold. Moreover, assume that there exist two continuous functions $\alpha: \mathbb{R}^{+} \rightarrow \mathbb{R}^{+}$and $\beta: J(M) \rightarrow(0, \infty)$, and a real constant $a_{0}>M_{0} f(0)$ satisfying conditions (4.36) and (4.37) of Theorem 4.2.

Then the solutions $u\left(t, t_{0}, u_{0}, \dot{u}_{0}\right)$ of (1.1) such that $V\left(t_{0}, u_{0}, \dot{u}_{0}\right)<a_{0} / M_{0}$ exist globally in the future and are $z$-bounded. 
Moreover, if (5.1) is replaced by condition (iv) of Theorem 5.1, then the solutions $u\left(t, t_{0}, u_{0}, \dot{u}_{0}\right)$ of $(1.1)$ such that $V\left(t_{0}, u_{0}, \dot{u}_{0}\right)<a_{0} / M_{0}$ exist globally in the future and are $(x, p)$-bounded.

Proof. We omit the proof since it is parallel to that of Theorem 3.2. It is enough to note that if $v(t)=V(t, u(t), \dot{u}(t))$ and $v\left(t_{0}\right)<a_{0} / M_{0}$, the condition (4.37) of Theorem 4.2 ensures that the solution $w\left(t, t_{0}, v\left(t_{0}\right)\right)$ of the comparison equation

$$
\dot{w}=\frac{\delta(t) M(t)}{\beta(M(t))} \alpha(w)
$$

is bounded on $\left[t_{0}, \infty\right)$.

CASE 2. Assume that there exists a continuous function $\rho: \mathbb{R}^{+} \rightarrow \mathbb{R}$, with $\int^{\infty} \rho(s) d s<$ $\infty$, such that

$$
\begin{aligned}
& (Q(t, u, p), p)+F_{t}(t, u) \\
& \quad \leq \rho(t) \psi(V)-2 \dot{\sigma}(t)|x| f^{\prime}(\sigma(t)|x|)-a(t) D(t, x, y) \quad \text { on } \mathbb{R}^{+} \times \mathbb{R}^{N} \times \mathbb{R}^{N},
\end{aligned}
$$

and denote by $J(P)$ the range of the function $P(t)$ defined by (2.52).

Fix $t_{0} \geq 0$ and put $P_{0}=P\left(t_{0}\right)$.

THEOREM 5.3. Suppose that conditions (4.1), (4.4), (5.1), and (5.24) hold. Moreover, assume that there exist two continuous functions $\tilde{\alpha}: \mathbb{R}^{+} \rightarrow \mathbb{R}^{+}$and $\tilde{\beta}: J(P) \rightarrow(0, \infty)$, and a real constant $b_{0}>0$ satisfying conditions (4.44) and (4.45) of Theorem 4.3.

Then the solutions $u\left(t, t_{0}, u_{0}, \dot{u}_{0}\right)$ of (1.1) such that $V\left(t_{0}, u_{0}, \dot{u}_{0}\right)<f\left(b_{0} / P_{0}\right)$ exist globally in the future and are $z$-bounded.

Moreover, if (5.1) is replaced by condition (iv) of Theorem 5.1, then the solutions $u\left(t, t_{0}, u_{0}, \dot{u}_{0}\right)$ of (1.1) such that $V\left(t_{0}, u_{0}, \dot{u}_{0}\right)<f\left(b_{0} / P_{0}\right)$ exist globally in the future and are $(x, p)$-bounded.

Proof. We omit the proof since it is parallel to that of Theorem 3.3.

Theorems 5.2 and 5.3 have the following corollaries. Their proofs are essentially the same as those of Corollaries 2.2 and 2.3, respectively.

COROLLARY 5.2. Fix $t_{0} \geq 0$ and assume that there is a function $\delta(t)$ satisfying (4.12) such that $\int_{t_{0}}^{\infty} \delta(t) d t<\int_{0}^{\infty} d s / g(s)$. Moreover, suppose that (4.1), (4.4), (5.1), and condition (4.51) of Corollary 4.2 hold.

If $r_{0}>0$ is the real constant satisfying (4.52), then the solutions $u\left(t, t_{0}, u_{0}, \dot{u}_{0}\right)$ of (1.1) such that $V\left(t_{0}, u_{0}, \dot{u}_{0}\right)<f\left(r_{0}\right)$ exist globally in the future and are $z$-bounded.

Moreover, if (5.1) is replaced by condition (iv) of Theorem 5.1, then the solutions $u\left(t, t_{0}, u_{0}, \dot{u}_{0}\right)$ of (1.1) such that $V\left(t_{0}, u_{0}, \dot{u}_{0}\right)<f\left(r_{0}\right)$ exist globally in the future and are $(x, p)$-bounded.

COROLlary 5.3. Let $h \geq 0, k>1$ and $n>h+k$ be real constants, and put $m=$ $(n-h-1) /(k-1)$. Suppose that conditions (4.4) and (5.1) hold. Moreover, assume that there exist a continuous function $\rho: \mathbb{R}^{+} \rightarrow \mathbb{R}^{+}$, of class $L^{1}\left(\mathbb{R}^{+}\right)$, and a continuous function $b: \mathbb{R}^{+} \rightarrow(0, \infty)$ satisfying conditions (4.44) and (4.45) of Theorem 4.3. 
Let $\delta$ be the function defined by (4.55). If $\delta P^{1-m} \in L^{1}\left(\mathbb{R}^{+}\right)$, then the solutions $u\left(t, t_{0}\right.$, $\left.u_{0}, \dot{u}_{0}\right)$ of (1.1) such that

$$
\begin{aligned}
H\left(u_{0}, \dot{u}_{0}\right)+F & \left(t_{0}, u_{0}\right)+2\left(\sigma\left(t_{0}\right)\left|x_{0}\right|\right)^{n} \\
& <P_{0}^{-n}\left[(m-1) \int_{t_{0}}^{\infty} \delta(t)(P(t))^{1-m} d t\right]^{n /(1-m)}
\end{aligned}
$$

exists globally in the future and $z$-bounded.

Finally, if (5.1) is replaced by condition (iv) of Theorem 5.1, then the above-mentioned solutions of (1.1) exist globally in the future and are $(x, p)$-bounded.

6. Examples and concluding remarks. The purpose of this section is to illustrate, by means of examples, some of the criteria previously established and to suggest various extensions.

EXAMPLE 1. Consider the scalar differential equation

$$
\ddot{u}-\frac{u \dot{u}^{2}}{1+u^{2}}+a(t) \dot{u}-b(t) u=0
$$

where $a: \mathbb{R}^{+} \rightarrow \mathbb{R}$ is a continuous function and $b: \mathbb{R}^{+} \rightarrow(0, \infty)$ is a function of class $C^{1}$. Equation (6.1) corresponds to

$$
H(p)=\frac{1}{2} p^{2}, \quad F(t, u)=-\frac{1}{2} b(t) u^{2}, \quad Q(t, u, p)=\frac{u}{1+u^{2}} p^{2}-a(t) p .
$$

Thus, conditions (2.1) and (2.2) are satisfied by taking $h(s)=(1 / 2) s^{2}, \tau(s)=s, f(s)=$ $(1 / 2) s^{2}$ and $\sigma(t)=\sqrt{b(t)}$. On the contrary, observe that condition (i) and (ii) of Theorem 2.1 cannot be satisfied.

To avoid this obstacle, we observe that one can choose the functions $H, F$, and $Q$ in a different way. In fact, equation (6.1) corresponds to the case

$$
H(u, p)=\frac{1}{2} \frac{p^{2}}{1+u^{2}}, \quad F(t, u)=-\frac{1}{2} b(t) \log \left(1+u^{2}\right), \quad Q(t, u, p)=-a(t) \frac{p}{1+u^{2}} .
$$

Since

$$
H(u, p) \geq \frac{1}{8}\left(\frac{2|u|}{1+u^{2}}|p|\right)^{2} \quad \text { on } \mathbb{R} \times \mathbb{R}
$$

conditions (2.1) and (2.2) are satisfied by $h(s)=(1 / 8) s^{2}, \tau(s)=\log \left(1+s^{2}\right), f(s)=$ $(1 / 2) s$, and $\sigma(t)=b(t)$. Moreover, condition (i) of Theorem 2.1 holds if $\mu(t)=0$ and $\rho(t)=\max _{t \in \mathbb{R}^{+}}\{2|a(t)|,|\dot{b}(t)| / b(t)\}$ (note that $\psi(s)=s$ ).

Then from Theorem 2.1, we see that all the solutions of equation (6.1) exist globally in the future.

EXAMPLE 2. Consider the scalar differential equation

$$
4 \dot{u}^{2} \ddot{u}+\dot{u}^{4}+e^{-t} e^{-u} \frac{\dot{u}}{1+u^{2}}-\frac{u e^{-u}}{1+u^{2}}=0,
$$


which corresponds to

$$
H(u, p)=e^{u} p^{4}, \quad F(u)=-\frac{1}{2} \log \left(1+u^{2}\right), \quad Q(t, u, p)=-e^{-t} \frac{p}{1+u^{2}} .
$$

First of all, we observe that

$$
H(u, p) \geq e^{-|u|} p^{4} \text { on } \mathbb{R} \times \mathbb{R} .
$$

Consequently, inequality (2.2) holds if $h(s)=s^{4}$ and $\tau(s)=4\left(1-e^{-s / 4}\right)$. Therefore, since the function $\tau$ is bounded, we are unable to use the criteria established in Sections 2 and 3.

We thus turn our attention to the results of Sections 4 and 5. Condition (4.1) holds with $f(s)=(1 / 2) \log \left(1+s^{2}\right)$ and $\sigma(t) \equiv 1$, and $s f^{\prime}(s)$ is an increasing function (it is worth noting that $f$ is not convex).

Moreover, since

$$
\begin{aligned}
& (Q,(t, u, p), p)+F_{t}(t, u) \\
& \quad=-e^{-t} \frac{p^{2}}{1+u^{2}}=-\frac{e^{-t}}{2} \frac{2|u|}{1+u^{2}}|u|\left(\frac{|p|}{|u|}\right)^{2} \quad \text { on } \mathbb{R}^{+} \times \mathbb{R} \times \mathbb{R},
\end{aligned}
$$

condition (4.10) of Theorem 4.1 is satisfied by $\mu(t)=\rho(t) \equiv 0, a(t)=e^{-t} / 2, g(s)=s$, and $\Gamma(s)=s^{2}$. Furthermore, $g(s) f^{\prime}(s)$ is an increasing function.

Then by Theorem 4.1, we see that all the solutions of (6.5) exist globally in the future.

EXAMPLE 3. Consider the following system of two scalar equations,

$$
\begin{gathered}
4 \dot{u}_{1}^{2} \ddot{u}_{1}+\dot{u}_{1}^{4}+\frac{1}{2} e^{t} e^{-u_{1}} \frac{\dot{u}_{1}}{\left(1+u_{1}^{2}\right)^{2}}-\frac{u_{1} e^{-u_{1}}}{1+u_{1}^{2}}+u_{2}=0 \\
\ddot{u}_{2}+\dot{u}_{1} \dot{u}_{2}+u_{2}=0
\end{gathered}
$$

corresponding to

$$
\begin{aligned}
H & =e^{u_{1}}\left(p_{1}^{4}+p_{2}^{2}\right), \quad F=e^{u_{1}} u_{2}^{2}-\log \left(1+u_{1}^{2}\right), \\
Q_{1} & =-\frac{1}{2} e^{t} \frac{p_{1}}{\left(1+u_{1}^{2}\right)^{2}}, \quad Q_{2}=0 .
\end{aligned}
$$

Conditions (4.1) and (4.4) hold by taking $f(s)=(1 / 2) \log \left(1+s^{2}\right), \sigma(t) \equiv 1, x=u_{1}$, and

$$
\Phi_{\omega}= \begin{cases}\frac{1}{2} e^{-\omega} s^{2}, & 0 \leq s \leq 1 \\ \frac{1}{2} e^{-\omega} s, & s \geq 1\end{cases}
$$

Moreover, since

$$
(Q(t, u, p), p)+F_{t}(t, u) \leq-\frac{1}{2} e^{t} \frac{\left|u_{1}\right|}{1+u_{1}^{2}}\left(1+u_{1}^{2}\right)^{3 / 2}\left(\frac{\left|p_{1}\right|}{\left(1+u_{1}^{2}\right)^{3 / 2}}\right)^{2}
$$

on $\mathbb{R}^{+} \times \mathbb{R}^{2} \times \mathbb{R}^{2}$, we see that condition (4.51) of Corollary 4.2 is satisfied with $g(s)=$ $\left(1+s^{2}\right)^{3 / 2}$ and $\Gamma(s)=s^{2}$. Consequently, in view of (4.13), we have $\delta(t)=2 e^{-t}$.

Finally, an easy calculation shows that if $t_{0} \geq \log 2$, then the constant $r_{0}$ satisfying (4.52) is given by $r_{0}=(1 / 2)\left(e^{t_{0}}-2\right) / \sqrt{e^{t_{0}}-1}$.

Then, from Corollary 4.2, we see that the solutions of (6.9), whose initial conditions $u_{0}=\left(u_{01}, u_{02}\right)$ and $\dot{u}_{0}=\left(\dot{u}_{01}, \dot{u}_{02}\right)$ satisfy the inequality 


$$
e^{u_{01}}\left(\dot{u}_{01}^{4}+\dot{u}_{02}^{2}\right)+e^{u_{01}} u_{02}^{2}+\frac{1}{2} \log \left(1+u_{01}^{2}\right) \leq t_{0}-\log 2-\frac{1}{2} \log \left(e^{t_{0}}-1\right),
$$

exist globally in the future.

Furthermore, assume that $t_{0}>\log 2$. Then, by Corollary 5.2 , we see that the solutions $u\left(t, t_{0}, u_{0}, \dot{u}_{0}\right)$ of (6.9) such that

$$
e^{u_{01}}\left(\dot{u}_{01}^{4}+\dot{u}_{02}^{2}\right)+e^{u_{02}} u_{02}^{2}+\frac{1}{2} \log \left(1+u_{01}^{2}\right)<t_{0}-\log 2-\frac{1}{2} \log \left(e^{t_{0}}-1\right)
$$

exist globally in the future and are $\left(u_{1}, p_{1}, p_{2}\right)$-bounded.

EXAMPLE 4. Consider the scalar differential equation

$$
(2+|\dot{u}|) e^{|\dot{u}|} \ddot{u}-\dot{u}-e^{t} e^{u}=0,
$$

corresponding to

$$
H(p)=p^{2} e^{|p|}, \quad F(t, u)=-e^{t} e^{u}, \quad Q(p)=p .
$$

We see that condition (2.2) is satisfied by taking $h(s)=s^{2} e^{s}$ and $\tau(s)=s$, whereas in place of (2.1), we have the condition

$$
F(t, u) \geq(1-\sigma(t)) f(\tau(|x|)) \quad \text { on } \mathbb{R}^{+} \times \mathbb{R}^{N},
$$

with $f(s)=e^{s}, \sigma(t)=e^{t}+1$ and $x=u$.

To deal with this case, it is necessary to modify the criteria established in Sections 2 and 3. This is possible by choosing as Liapunov function

$$
V(t, u, p)=H(u, p)+F(t, u)+\sigma(t) f(\tau(|x|)) .
$$

Alike, if (4.2) is satisfied together with a condition of the type

$$
F(t, u) \geq(1-\sigma(t)) f(|x|) \text { on } \mathbb{R}^{+} \times \mathbb{R}^{N},
$$

then we modify the criteria established in Sections 4 and 5 by introducing as Liapunov function

$$
V(t, u, p)=H(u, p)+F(t, u)+\sigma(t) f(|x|) .
$$

We limit ourselves to stating the following two Theorems, which are natural extensions of Theorems 2.1 and 4.1, respectively. Corresponding results can also be obtained extending the remaining criteria previously established, but we leave their statement to the interested reader.

THEOREM 6.1. Suppose that conditions (2.2), (2.3), and (6.18) hold. Moreover, assume that there exist two continuous functions $\mu: \mathbb{R}^{+} \rightarrow \mathbb{R}$ and $\phi: \mathbb{R}^{+} \rightarrow \mathbb{R}^{+}$such that

$$
\begin{gathered}
(Q(t, u, p), p)+F_{t}(t, u) \leq \mu(t) \phi(V) \quad \text { on } \mathbb{R}^{+} \times \mathbb{R}^{N} \times \mathbb{R}^{N}, \\
\int^{\infty} \frac{d s}{f(s) / f^{\prime}(s)+(\phi \circ f)(s) / f^{\prime}(s)+\left(h^{-1} \circ f\right)(s)}=\infty .
\end{gathered}
$$

Then all solutions of (1.1) exist globally in the future. 
THEOREM 6.2. Suppose that conditions (4.4) and (6.20) hold. Moreover, assume that there exist two continuous functions $\mu: \mathbb{R}^{+} \rightarrow \mathbb{R}$ and $\phi: \mathbb{R}^{+} \rightarrow \mathbb{R}^{+}$such that

$$
\begin{gathered}
(Q(t, u, p), p)+F_{t}(t, u) \leq \mu(t) \phi(V)-a(t) D(x, y) \quad \text { on } \mathbb{R}^{+} \times \mathbb{R}^{N} \times \mathbb{R}^{N}, \\
\int^{\infty} \frac{d s}{f(s) / f^{\prime}(s)+(\phi \circ f)(s) / f^{\prime}(s)+g(s)}=\infty .
\end{gathered}
$$

where here it is

$$
D(x, y)=f^{\prime}(|x|) g(|x|) \Gamma\left(\frac{|y|}{g(|x|)}\right) .
$$

Then all solutions of (1.1) exist globally in the future.

REMARK 6.1. Theorem 6.1 improves [3, Thm. 1], where $H=H(p)$ and $(Q(t, u, p), p)$ $\leq 0$ on $\mathbb{R}^{+} \times \mathbb{R}^{N} \times \mathbb{R}^{N}$. To avoid any misunderstanding, it is worth noting that when $H=H(p)$, the function $\tau$ introduced in [3] coincides with the function $f$ in (6.18).

Now, coming back to Example 4, we see that

$$
(Q(p), p)+F_{t}(t, u) \leq p^{2} e^{|p|}+e^{t} e^{|u|} \quad \text { on } \mathbb{R}^{+} \times \mathbb{R} \times \mathbb{R},
$$

and, thus, condition (6.22) of Theorem 6.1 holds by taking $\mu(t) \equiv 1$ and $\phi(s)=s$. Finally, since $s^{2} e^{s} \geq e^{s}$ on $[1, \infty)$, it follows that

$$
\left(h^{-1} \circ f\right)(s) \leq s \text { on }[1, \infty),
$$

and, thus, condition (6.23) of Theorem 6.1 holds as well.

ACKNOWLEDgement. The author wishes to thank Professor J. Serrin for a number of helpful suggestions. The author is a member of Gruppo Nazionale per la Fisica Matematica of the Consiglio Nazionale delle Ricerche. This research has been supported by the Italian Ministero della Università e della Ricerca Scientifica e Tecnologica.

\section{REFERENCES}

[1] G. Cantarelli, Stabilization of the equilibrium of holonomic systems by means of timedependent dissipative forces, Boll. Un. Mat. Ital. A (7) 6 (1992), no. 2, 193-196 (Italian). MR 93f:70024. Zbl 758.70008.

[2] _ New criteria for future global existence of motions of scleronomic systems, Ann. Mat. Pura Appl. (4) 163 (1993), 247-264 (Italian). MR 94d:34014. Zbl 771.70011.

[3] _ Global existence of solutions of second order differential systems, Asymptotic Anal. 8 (1994), no. 1, 93-101. MR 95b:34002. Zbl 795.34001.

[4]__ Global existence in the future and boundedness of submanifolds of solutions of a scalar comparison equation, Rend. Circ. Mat. Palermo (2) 44 (1995), no. 3, 401-416. MR 97b:34004. Zbl 858.34002.

[5]___ Global existence of submanifolds of solutions of nonlinear second order differential systems, Differential Integral Equations 8 (1995), no. 6, 1385-1394. MR 96h:34084. Zbl 824.34004 . 
[6] G. Cantarelli and C. Risito, Criteria of global existence and boundedness for scleronomic holonomic systems, Ann. Mat. Pura Appl. (4) 162 (1992), 383-394 (Italian). MR 93m:34022. Zbl 768.70010.

[7] R. Conti, Limitazioni "in ampiezza" delle soluzioni di un sistema di equazioni differenziali e applicazioni, Boll. Un. Mat. Ital. (3) 11 (1956), 344-349 (Italian). MR 18,309c. Zbl 071.30601.

[8]__ Sulla prolungabilitá delle soluzioni di un sistema di equazioni differenziali ordinarie, Boll. Un. Mat. Ital. (3) 11 (1956), 510-514 (Italian). MR 18,736e. Zbl 072.30403 .

[9] P. Pucci and J. Serrin, Continuation and limit properties for solutions of strongly nonlinear second order differential equations, Asymptotic Anal. 4 (1991), no. 2, 97-160. MR 92j:34069. Zbl 733.34042.

[10] C Continuation and limit behavior for damped quasi-variational systems, Degenerate diffusions (Minneapolis, MN, 1991) (New York) (W. M. Ni, L. A. Peletier, and J. L. Vasquez, eds.), IMA Vol. Math. Appl., vol. 47, Springer, 1993, pp. 157-173. MR 95g:34006. Zbl 796.34023.

[11] On the derivation of Hamilton's equations, Arch. Rational Mech. Anal. 125 (1994), no. 4, 297-310. MR 94m:58090. Zbl 809.70012.

[12] C. Risito, Global existence and boundedness of the motions of holonomic scleronomic systems, Ann. Mat. Pura Appl. (4) 139 (1985), 341-348 (Italian). MR 87g:70025. Zbl 568.70012.

[13] T. Yoshizawa, Stability theory by Liapunov's second method, no. 9, The Mathematical Society of Japan, Tokyo, 1966. MR 34\#7896. Zbl 144.10802.

CANTARelli: Dipartimento di Matematica, Università degli studi di PARMa, Via M. D’AZEGLIO 85/A - 43100 PARMA, ITALY 


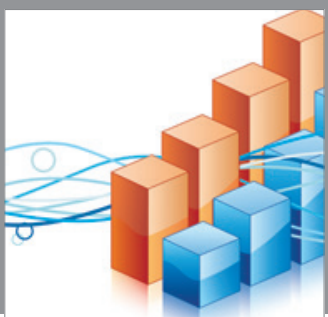

Advances in

Operations Research

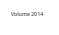

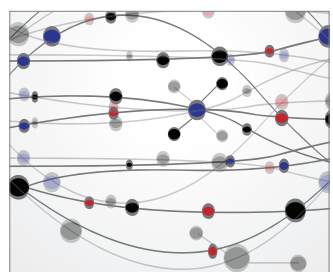

\section{The Scientific} World Journal
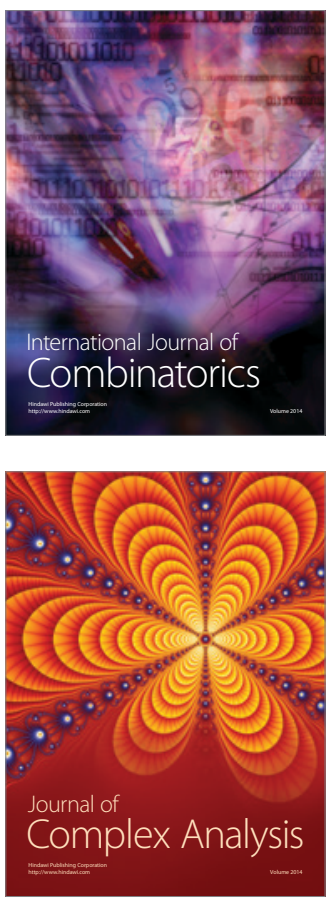

International Journal of

Mathematics and

Mathematical

Sciences
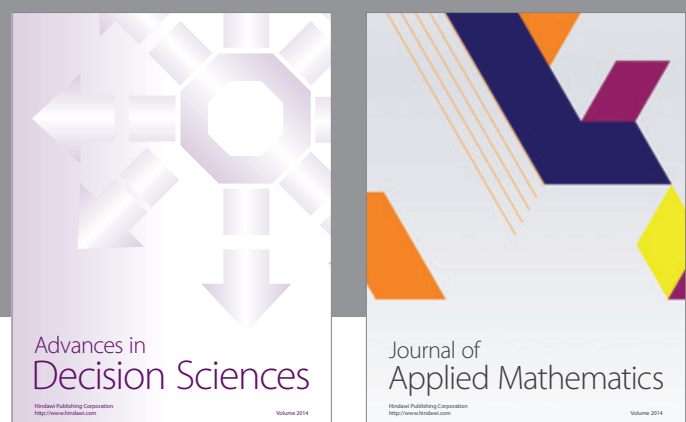

Journal of

Applied Mathematics
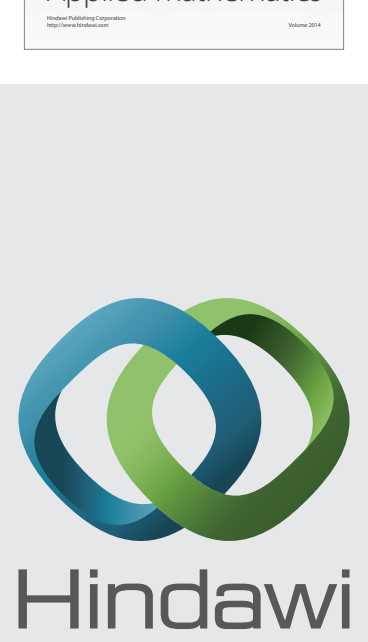

Submit your manuscripts at http://www.hindawi.com
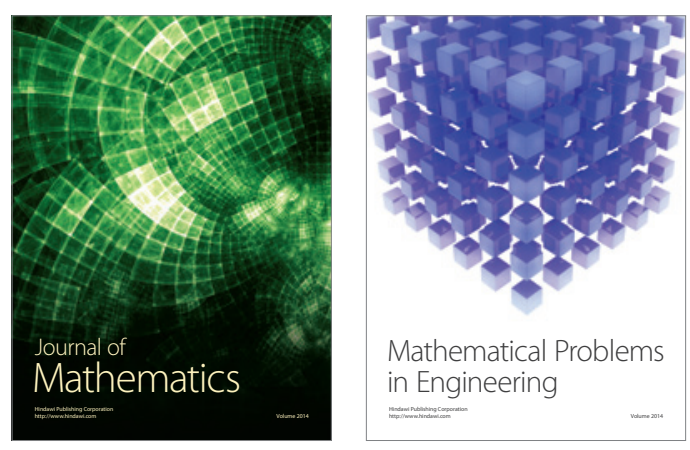

Mathematical Problems in Engineering
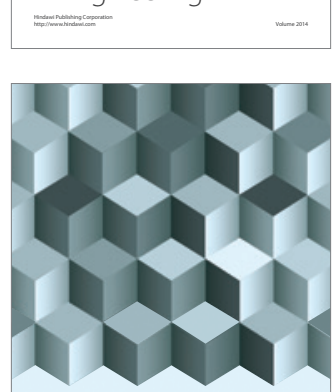

Journal of

Function Spaces
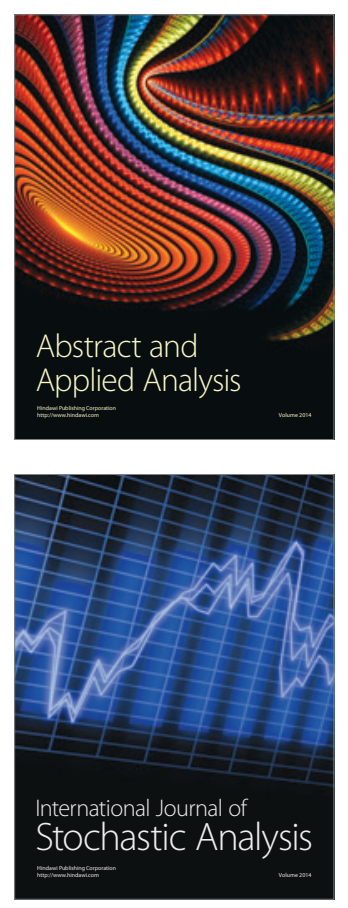

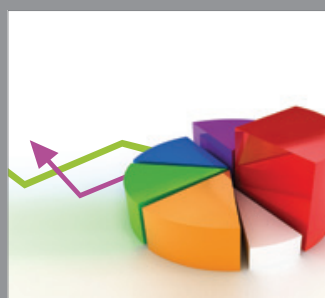

ournal of

Probability and Statistics

Promensencen
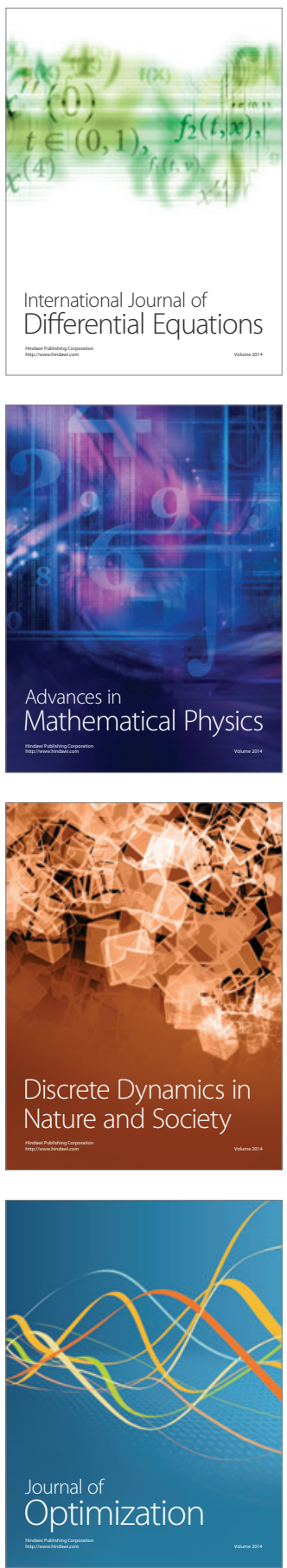